\title{
Evolution of Specialization and Ecological Character Displacement of Herbivores along a Gradient of Plant Quality
}

Martijn Egas (egas@science.uva.nl)

Maurice W. Sabelis (sabelis@ science.uva.nl)

Ulf Dieckmann (dieckmann@iiasa.ac.at)

\section{Approved by}

Leen Hordijk

Director, IIASA

March 2005 Institute, its National Member Organizations, or other organizations supporting the work. 


\section{IIASA STUdIES IN ADAPTIVE DYNAMICS No. 93}

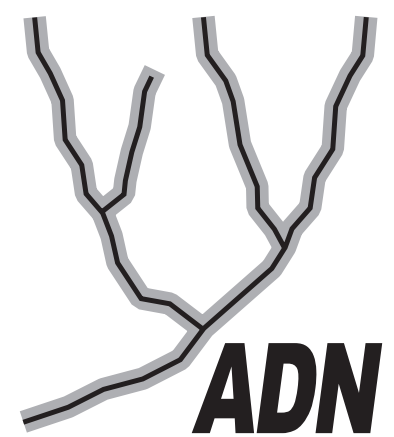

The Adaptive Dynamics Network at IIASA fosters the development of new mathematical and conceptual techniques for understanding the evolution of complex adaptive systems.

Focusing on these long-term implications of adaptive processes in systems of limited growth, the Adaptive Dynamics Network brings together scientists and institutions from around the world with IIASA acting as the central node.

Scientific progress within the network is collected in the IIASA Studies in Adaptive Dynamics series.

No. 1 Metz JAJ, Geritz SAH, Meszéna G, Jacobs FJA, van Heerwaarden JS: Adaptive Dynamics: A Geometrical Study of the Consequences of Nearly Faithful Reproduction. IIASA Working Paper WP-95-099 (1995). van Strien SJ, Verduyn Lunel SM (eds): Stochastic and Spatial Structures of Dynamical Systems, Proceedings of the Royal Dutch Academy of Science (KNAW Verhandelingen), North Holland, Amsterdam, pp. 183-231 (1996).

No. 2 Dieckmann U, Law R: The Dynamical Theory of Coevolution: A Derivation from Stochastic Ecological Processes. IIASA Working Paper WP-96-001 (1996). Journal of Mathematical Biology 34:579-612 (1996).

No. 3 Dieckmann U, Marrow P, Law R: Evolutionary Cycling of Predator-PreyInteractions: Population Dynamics and the Red Queen. IIASA Preprint (1995). Journal of Theoretical Biology 176:91-102 (1995).

No. 4 Marrow P, Dieckmann U, Law R: Evolutionary Dynamics of Predator-Prey Systems: An Ecological Perspective. IIASA Working Paper WP-96-002 (1996). Journal of Mathematical Biology 34:556-578 (1996).

No. 5 Law R, Marrow P, Dieckmann U: On Evolution under Asymmetric Competition. IIASA Working Paper WP-96-003 (1996). Evolutionary Ecology 11:485-501 (1997).

No. 6 Metz JAJ, Mylius SD, Diekmann O: When Does Evolution Optimize? On the Relation Between Types of Density Dependence and Evolutionarily Stable Life History Parameters. IIASA Working Paper WP-96-004 (1996).

No. 7 Ferrière R, Gatto M: Lyapunov Exponents and the Mathematics of Invasion in Oscillatory or Chaotic Populations. Theoretical Population Biology 48:126-171 (1995).

No. 8 Ferrière R, Fox GA: Chaos and Evolution. IIASA Preprint (1996). Trends in Ecology and Evolution 10:480485 (1995).

No. 9 Ferrière R, Michod RE: The Evolution of Cooperation in Spatially Heterogeneous Populations. IIASA Working Paper WP-96-029 (1996). The American Naturalist 147:692717 (1996).

No. 10 van Dooren TJM, Metz JAJ: Delayed Maturation in Temporally Structured Populations with Non-Equilibrium Dynamics. IIASA Working Paper WP-96-070 (1996). Journal of Evolutionary Biology 11:41-62 (1998).
No. 11 Geritz SAH, Metz JAJ, Kisdi É, Meszéna G: The Dynamics of Adaptation and Evolutionary Branching. IIASA Working Paper WP-96-077 (1996). Physical Review Letters 78:2024-2027 (1997).

No. 12 Geritz SAH, Kisdi É, Meszéna G, Metz JAJ: Evolutionary Singular Strategies and the Adaptive Growth and Branching of the Evolutionary Tree. IIASA Working Paper WP-96-114 (1996). Evolutionary Ecology 12:35-57 (1998).

No. 13 Heino M, Metz JAJ, Kaitala V: Evolution of Mixed Maturation Strategies in Semelparous Life-Histories: The Crucial Role of Dimensionality of Feedback Environment. IIASA Working Paper WP-96-126 (1996). Philosophical Transactions of the Royal Society of London Series B 352:1647-1655 (1997).

No. 14 Dieckmann U: Can Adaptive Dynamics Invade? IIASA Working Paper WP-96-152 (1996). Trends in Ecology and Evolution 12:128-131 (1997).

No. 15 Meszéna G, Czibula I, Geritz SAH: Adaptive Dynamics in a 2-Patch Environment: A Simple Model for Allopatric and Parapatric Speciation. IIASA Interim Report IR-97-001 (1997). Journal of Biological Systems 5:265-284 (1997).

No. 16 Heino M, Metz JAJ, Kaitala V: The Enigma of Frequency-Dependent Selection. IIASA Interim Report IR97-061 (1997). Trends in Ecology and Evolution 13:367-370 (1998).

No. 17 Heino M: Management of Evolving Fish Stocks. IIASA Interim Report IR-97-062 (1997). Canadian Journal of Fisheries and Aquatic Sciences 55:1971-1982 (1998).

No. 18 Heino M: Evolution of Mixed Reproductive Strategies in Simple Life-History Models. IIASA Interim Report IR-97063 (1997).

No. 19 Geritz SAH, van der Meijden E, Metz JAJ: Evolutionary Dynamics of Seed Size and Seedling Competitive Ability. IIASA Interim Report IR-97-071 (1997). Theoretical Population Biology 55:324-343 (1999).

No. 20 Galis F, Metz JAJ: Why Are There So Many Cichlid Species? On the Interplay of Speciation and Adaptive Radiation. IIASA Interim Report IR-97-072 (1997). Trends in Ecology and Evolution 13:1-2 (1998). 
No. 21 Boerlijst MC, Nowak MA, Sigmund K: Equal Pay for all Prisoners/ The Logic of Contrition. IIASA Interim Report IR-97-073 (1997). American Mathematical Society Monthly 104:303-307 (1997). Journal of Theoretical Biology 185:281-293 (1997).

No. 22 Law R, Dieckmann U: Symbiosis Without Mutualism and the Merger of Lineages in Evolution. IIASA Interim Report IR-97-074 (1997). Proceedings of the Royal Society of London Series B 265:1245-1253 (1998).

No. 23 Klinkhamer PGL, de Jong TJ, Metz JAJ: Sex and Size in Cosexual Plants. IIASA Interim Report IR-97-078 (1997). Trends in Ecology and Evolution 12:260-265 (1997).

No. 24 Fontana W, Schuster P: Shaping Space: The Possible and the Attainable in RNA Genotype-Phenotype Mapping. IIASA Interim Report IR-98-004 (1998). Journal of Theoretical Biology 194:491-515 (1998).

No. 25 Kisdi É, Geritz SAH: Adaptive Dynamics in Allele Space: Evolution of Genetic Polymorphism by Small Mutations in a Heterogeneous Environment. IIASA Interim Report IR-98-038 (1998). Evolution 53:993-1008 (1999).

No. 26 Fontana W, Schuster P: Continuity in Evolution: On the Nature of Transitions. IIASA Interim Report IR-98-039 (1998). Science 280:1451-1455 (1998).

No. 27 Nowak MA, Sigmund K: Evolution of Indirect Reciprocity by Image Scoring/ The Dynamics of Indirect Reciprocity. IIASA Interim Report IR-98-040 (1998). Nature 393:573-577 (1998). Journal of Theoretical Biology 194:561574 (1998).

No. 28 Kisdi É: Evolutionary Branching Under Asymmetric Competition. IIASA Interim Report IR-98-045 (1998). Journal of Theoretical Biology 197:149-162 (1999).

No. 29 Berger U: Best Response Adaptation for Role Games. IIASA Interim Report IR-98-086 (1998).

No. 30 van Dooren TJM: The Evolutionary Ecology of Dominance-Recessivity. IIASA Interim Report IR-98-096 (1998). Journal of Theoretical Biology 198:519-532 (1999).

No. 31 Dieckmann U, O'Hara B, Weisser W: The Evolutionary Ecology of Dispersal. IIASA Interim Report IR-98-108 (1998). Trends in Ecology and Evolution 14:88-90 (1999).

No. 32 Sigmund K: Complex Adaptive Systems and the Evolution of Reciprocation. IIASA Interim Report IR-98-100 (1998). Ecosystems 1:444-448 (1998).

No. 33 Posch M, Pichler A, Sigmund K: The Efficiency of Adapting Aspiration Levels. IIASA Interim Report IR-98103 (1998). Proceedings of the Royal Society London Series B 266:1427-1435 (1999).

No. 34 Mathias A, Kisdi É: Evolutionary Branching and Coexistence of Germination Strategies. IIASA Interim Report IR-99-014 (1999).

No. 35 Dieckmann U, Doebeli M: On the Origin of Species by Sympatric Speciation. IIASA Interim Report IR-99-013 (1999). Nature 400:354-357 (1999).

No. 36 Metz JAJ, Gyllenberg M: How Should We Define Fitness in Structured Metapopulation Models? Including an Application to the Calculation of Evolutionarily Stable Dispersal Strategies. IIASA Interim Report IR-99-019 (1999). Proceedings of the Royal Society of London Series B 268:499508 (2001)
No. 37 Gyllenberg M, Metz JAJ: On Fitness in Structured Metapopulations. IIASA Interim Report IR-99-037 (1999). Journal of Mathematical Biology 43:545-560 (2001).

No. 38 Meszéna G, Metz JAJ: Species Diversity and Population Regulation: The Importance of Environmental Feedback Dimensionality. IIASA Interim Report IR-99-045 (1999).

No. 39 Kisdi É, Geritz SAH: Evolutionary Branching and Sympatric Speciation in Diploid Populations. IIASA Interim Report IR-99-048 (1999).

No. 40 Ylikarjula J, Heino M, Dieckmann U: Ecology and Adaptation of Stunted Growth in Fish. IIASA Interim Report IR-99-050 (1999). Evolutionary Ecology 13:433-453 (1999).

No. 41 Nowak MA, Sigmund K: Games on Grids. IIASA Interim Report IR-99-038 (1999). Dieckmann U, Law R, Metz JAJ (eds): The Geometry of Ecological Interactions: Simplifying Spatial Complexity, Cambridge University Press, Cambridge, UK, pp. 135-150 (2000).

No. 42 Ferrière R, Michod RE: Wave Patterns in Spatial Games and the Evolution of Cooperation. IIASA Interim Report IR-99-041 (1999). Dieckmann U, Law R, Metz JAJ (eds): The Geometry of Ecological Interactions: Simplifying Spatial Complexity, Cambridge University Press, Cambridge, UK, pp. 318-332 (2000).

No. 43 Kisdi É, Jacobs FJA, Geritz SAH: Red Queen Evolution by Cycles of Evolutionary Branching and Extinction. IIASA Interim Report IR-00-030 (2000). Selection 2:161176 (2001).

No. 44 Meszéna G, Kisdi É, Dieckmann U, Geritz SAH, Metz JAJ: Evolutionary Optimisation Models and Matrix Games in the Unified Perspective of Adaptive Dynamics. IIASA Interim Report IR-00-039 (2000). Selection 2:193-210 (2001).

No. 45 Parvinen K, Dieckmann U, Gyllenberg M, Metz JAJ: Evolution of Dispersal in Metapopulations with Local Density Dependence and Demographic Stochasticity. IIASA Interim Report IR-00-035 (2000). Journal of Evolutionary Biology 16:143-153 (2003).

No. 46 Doebeli M, Dieckmann U: Evolutionary Branching and Sympatric Speciation Caused by Different Types of Ecological Interactions. IIASA Interim Report IR-00-040 (2000). The American Naturalist 156:S77-S101 (2000).

No. 47 Heino M, Hanski I: Evolution of Migration Rate in a Spatially Realistic Metapopulation Model. IIASA Interim Report IR-00-044 (2000). The American Naturalist 157:495511 (2001).

No. 48 Gyllenberg M, Parvinen K, Dieckmann U: Evolutionary Suicide and Evolution of Dispersal in Structured Metapopulations. IIASA Interim Report IR-00-056 (2000). Journal of Mathematical Biology 45:79-105 (2002).

No. 49 van Dooren TJM: The Evolutionary Dynamics of Direct Phenotypic Overdominance: Emergence Possible, Loss Probable. IIASA Interim Report IR-00-048 (2000). Evolution 54: 1899-1914 (2000).

No. 50 Nowak MA, Page KM, Sigmund K: Fairness Versus Reason in the Ultimatum Game. IIASA Interim Report IR00-57 (2000). Science 289:1773-1775 (2000).

No. 51 de Feo O, Ferrière R: Bifurcation Analysis of Population Invasion: On-Off Intermittency and Basin Riddling. IIASA Interim Report IR-00-074 (2000). International Journal of Bifurcation and Chaos 10:443-452 (2000). 
No. 52 Heino M, Laaka-Lindberg S: Clonal Dynamics and Evolution of Dormancy in the Leafy Hepatic Lophozia Silvicola. IIASA Interim Report IR-01-018 (2001). Oikos 94:525-532 (2001).

No. 53 Sigmund K, Hauert C, Nowak MA: Reward and Punishment in Minigames. IIASA Interim Report IR-01-031 (2001). Proceedings of the National Academy of Sciences of the USA 98:10757-10762(2001).

No. 54 Hauert C, De Monte S, Sigmund K, Hofbauer J: Oscillations in Optional Public Good Games. IIASA Interim Report IR-01-036 (2001).

No. 55 Ferrière R, Le Galliard J: Invasion Fitness and Adaptive Dynamics in Spatial Population Models. IIASA Interim Report IR-01-043 (2001). Clobert J, Dhondt A, Danchin E, Nichols J (eds): Dispersal, Oxford University Press, pp. 57-79 (2001).

No. 56 de Mazancourt C, Loreau M, Dieckmann U: Can the Evolution of Plant Defense Lead to Plant-Herbivore Mutualism. IIASA Interim Report IR-01-053 (2001). The American Naturalist 158: 109-123 (2001).

No. 57 Claessen D, Dieckmann U: Ontogenetic Niche Shifts and Evolutionary Branching in Size-Structured Populations. IIASA Interim Report IR-01-056 (2001). Evolutionary Ecology Research 4:189-217 (2002).

No. 58 Brandt H: Correlation Analysis of Fitness Landscapes. IIASA Interim Report IR-01-058 (2001).

No. 59 Dieckmann U: Adaptive Dynamics of Pathogen-Host Interacations. IIASA Interim Report IR-02-007 (2002). Dieckmann U, Metz JAJ, Sabelis MW, Sigmund K (eds): Adaptive Dynamics of Infectious Diseases: In Pursuit of Virulence Management, Cambridge University Press, Cambridge, UK, pp. 39-59 (2002).

No. 60 Nowak MA, Sigmund K: Super- and Coinfection: The Two Extremes. IIASA Interim Report IR-02-008 (2002). Dieckmann U, Metz JAJ, Sabelis MW, Sigmund K (eds): Adaptive Dynamics of Infectious Diseases: In Pursuit of Virulence Management, Cambridge University Press, Cambridge, UK, pp. 124-137 (2002).

No. 61 Sabelis MW, Metz JAJ: Perspectives for Virulence Management: Relating Theory to Experiment. IIASA Interim Report IR-02-009 (2002). Dieckmann U, Metz JAJ, Sabelis MW, Sigmund K (eds): Adaptive Dynamics of Infectious Diseases: In Pursuit of Virulence Management, Cambridge University Press, Cambridge, UK, pp. 379-398 (2002).

No. 62 Cheptou P, Dieckmann U: The Evolution of SelfFertilization in Density-Regulated Populations . IIASA Interim Report IR-02-024 (2002). Proceedings of the Royal Society of London Series B 269:1177-1186 (2002).

No. 63 Bürger R: Additive Genetic Variation Under Intraspecific Competition and Stabilizing Selection: A Two-Locus Study. IIASA Interim Report IR-02-013 (2002). Theoretical Population Biology 61:197-213 (2002).

No. 64 Hauert C, De Monte S, Hofbauer J, Sigmund K: Volunteering as Red Queen Mechanism for Co-operation in Public Goods Games. IIASA Interim Report IR-02-041 (2002). Science 296:1129-1132(2002).

No. 65 Dercole F, Ferrière R, Rinaldi S: Ecological Bistability and Evolutionary Reversals under Asymmetrical Competition. IIASA Interim Report IR-02-053 (2002). Evolution 56:1081-1090 (2002).
No. 66 Dercole F, Rinaldi S: Evolution of Cannibalistic Traits: Scenarios Derived from Adaptive Dynamics. IIASA Interim Report IR-02-054 (2002). Theoretical Population Biology 62:365-374 (2002).

No. 67 Bürger R, Gimelfarb A: Fluctuating Environments and the Role of Mutation in Maintaining Quantitative Genetic Variation. IIASA Interim Report IR-02-058 (2002). Genetical Research 80:31-46 (2002).

No. 68 Bürger R: On a Genetic Model of Intraspecific Competition and Stabilizing Selection. IIASA Interim Report IR02-062 (2002). Amer. Natur. 160:661-682 (2002).

No. 69 Doebeli M, Dieckmann U: Speciation Along Environmental Gradients. IIASA Interim Report IR-02-079 (2002). Nature 421:259-264 (2003).

No. 70 Dercole F, Irisson J, Rinaldi S: Bifurcation Analysis of a Prey-Predator Coevolution Model. IIASA Interim Report IR-02-078 (2002). SIAM Journal on Applied Mathematics 63:1378-1391 (2003).

No. 71 Le Galliard J, Ferrière R, Dieckmann U: The Adaptive Dynamics of Altruism in Spatially Heterogeneous Populations. IIASA Interim Report IR-03-006 (2003). Evolution 57:1-17 (2003).

No. 72 Taborsky B, Dieckmann U, Heino M: Unexpected Discontinuities in Life-History Evolution under SizeDependent Mortality. IIASA Interim Report IR-03-004 (2003). Proceedings of the Royal Society of London Series B 270:713-721 (2003).

No. 73 Gardmark A, Dieckmann U, Lundberg P: LifeHistory Evolution in Harvested Populations: The Role of Natural Predation. IIASA Interim Report IR-03-008 (2003). Evolutionary Ecology Research 5:239-257 (2003).

No. 74 Mizera F, Meszéna G: Spatial Niche Packing, Character Displacement and Adaptive Speciation Along an Environmental Gradient. IIASA Interim Report IR-03-062 (2003). Evolutionary Ecology Research 5: 363-382 (2003).

No. 75 Dercole F: Remarks on Branching-Extinction Evolutionary Cycles. IIASA Interim Report IR-03-075 (2003). Journal of Mathematical Biology 47: 569-580 (2003).

No. 76 Hofbauer J, Sigmund K: Evolutionary Game Dynamics. IIASA Interim Report IR-03-078 (2003). Bulletin of the American Mathematical Society 40: 479-519 (2003).

No. 77 Ernande B, Dieckmann U, Heino M: Adaptive Changes in Harvested Populations: Plasticity and Evolution of Age and Size at Maturation. IIASA Interim Report IR03-058 (2003). Proceedings of the Royal Society of London Series B-Biological Sciences, 271: 415-423 (2004).

No. 78 Hanski I, Heino M: Metapopulation-Level Adaptation of Insect Host Plant Preference and Extinction-Colonization Dynamics in Heterogeneous Landscapes. IIASA Interim Report IR-03-028 (2003). Theoretical Population Biology 63:309-338 (2003).

No. 79 van Doorn G, Dieckmann U, Weissing FJ: Sympatric Speciation by Sexual Selection: A Critical Re-Evaluation. IIASA Interim Report IR-04-003 (2004). American Naturalist 163: 709-725 (2004).

No. 80 Egas M, Dieckmann U, Sabelis MW: Evolution Restricts the Coexistence of Specialists and Generalists - the Role of Trade-off Structure. IIASA Interim Report IR-04-004 (2004). 
No. 81 Ernande B, Dieckmann U: The Evolution of Phenotypic Plasticity in Spatially Structured Environments: Implications of Intraspecific Competition, Plasticity Costs, and Environmental Characteristics. IIASA Interim Report IR-04-006 (2004). Journal of Evolutionary Biology 17 (3): 613-628 (2004).

No. 82 Cressman R, Hofbauer J: Measure Dynamics on a One-Dimensional Continuous Trait Space: Theoretical Foundations for Adaptive Dynamics. IIASA Interim Report IR04-016 (2004).

No. 83 Cressman R: Dynamic Stability of the Replicator Equation with Continuous Strategy Space. IIASA Interim Report IR-04-017 (2004).

No. 84 Ravigné V, Olivieri I, Dieckmann U: Implications of Habitat Choice for Protected Polymorphisms. IIASA Interim Report IR-04-005 (2004). Evolutionary Ecology Research 6: 125-145 (2004).

No. 85 Nowak MA, Sigmund K: Evolutionary Dynamics of Biological Games. IIASA Interim Report IR-04-013 (2004). Science 303: 793-799 (2004).

No. 86 Vukics A, Asbóth J, Meszéna G: Speciation in Multidimensional Evolutionary Space. IIASA Interim Report IR-04-028 (2004). Physical Review E 684 (2003).
No. 87 de Mazancourt C, Dieckmann U: Trade-off Geometries and Frequency-dependent Selection. IIASA Interim Report IR-04-039 (2004).

No. 88 Cadet CR, Metz JAJ, Klinkhamer PGL: Size and the Not-So-Single Sex: disentangling the effects of size on sex allocation. IIASA Interim Report IR-04-084 (2004). American Naturalist, 164: 779-792 (2004).

No. 89 Rueffler C, van Dooren TJM, Metz JAJ: Adaptive Walks on Changing Landscapes: Levins' Approach Extended. IIASA Interim Report IR-04-083 (2004). Theoretical Population Biology, 65: 165-178 (2004).

No. 90 de Mazancourt C, Loreau M, Dieckmann U: Understanding Mutualism When There is Adaptation to the Partner. IIASA Interim Report IR-05-016 (2005).

No. 91 Dieckmann U, Doebeli M: Pluralism in Evolutionary Theory. IIASA Interim Report IR-05-017 (2005).

No. 92 Doebeli M, Dieckmann U, Metz JAJ, Tautz D: What We Have Also Learned. IIASA Interim Report IR-05-018 (2005).

No. 93 Egas M, Sabelis MW, Dieckmann U: Evolution of Specialization and Ecological Character Displacement of Herbivores Along a Gradient of Plant Quality. IIASA Interim Report IR-05-019 (2005).

Issues of the IIASA Studies in Adaptive Dynamics series can be obtained at www.iiasa.ac.at/Research/ADN/Series.html or by writing to adn@iiasa.ac.at. 


\section{Contents}

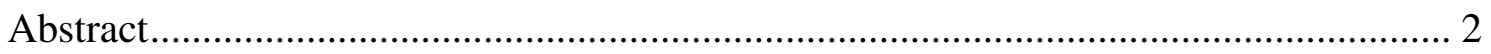

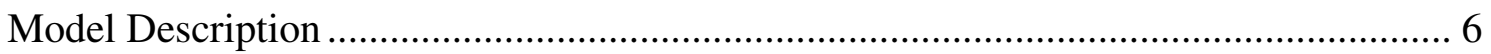

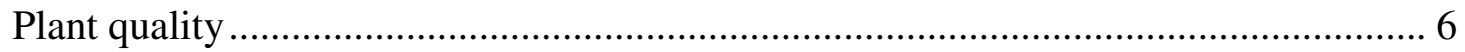

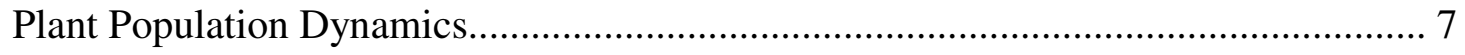

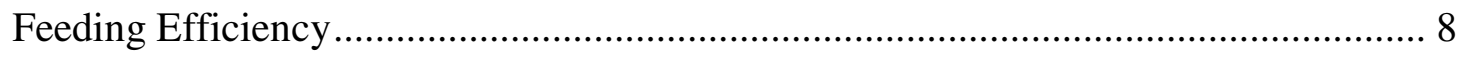

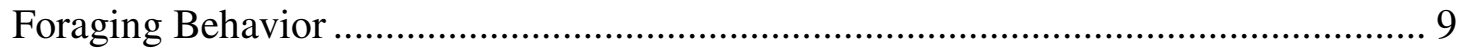

Herbivore Population Dynamics............................................................................. 11

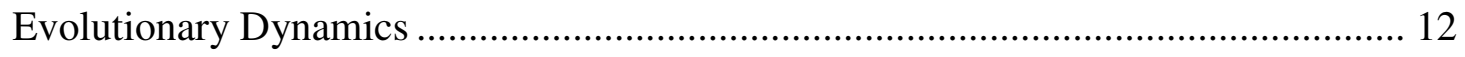

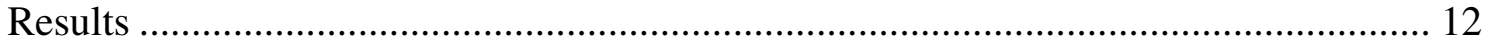

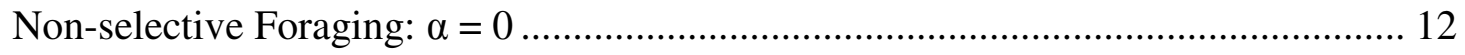

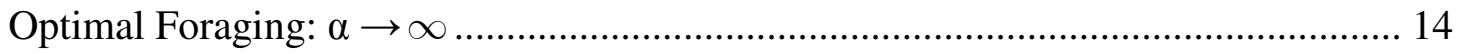

Sub-optimal Foraging - Deterministic Approximation........................................... 15

Sub-optimal Foraging - Individual-based Model ...................................................... 17

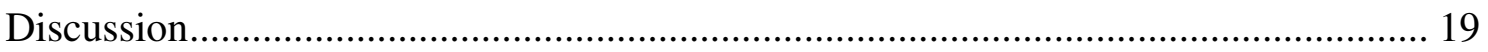

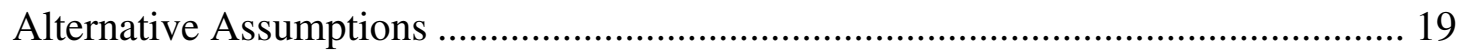

The Interplay between Ecological Character Displacement and Specialization ......... 21

Limiting Similarity and the Evolution of Specialization .......................................... 22

Ecological Character Displacement.......................................................................... 24

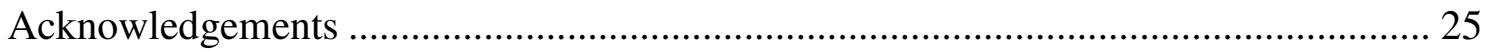

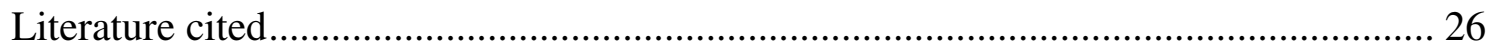

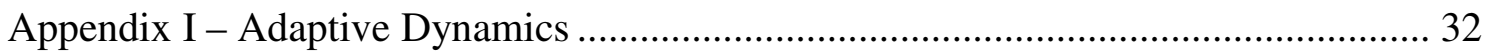

Appendix II - Non-selective Foraging: $\alpha=0$...................................................... 34

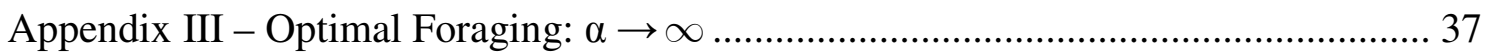

Appendix IV - Sub-optimal Foraging: $0<\alpha<\infty$..................................................... 39

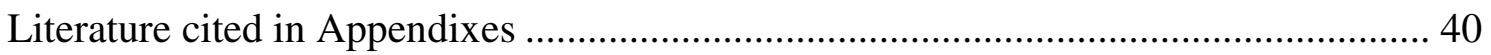

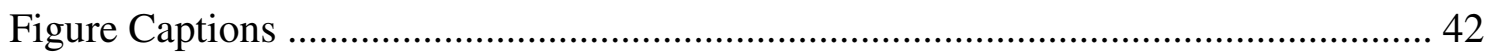

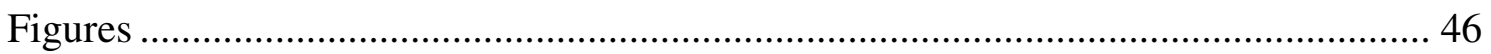




\title{
EVOLUTION OF SPECIALIZATION AND ECOLOGICAL CHARACTER DISPLACEMENT OF HERBIVORES ALONG A GRADIENT OF PLANT QUALITY
}

\author{
Martijn Egas ${ }^{1,2, *}$, Maurice W. Sabelis, ${ }^{1, \pi}$, and Ulf Dieckmann ${ }^{2, \S}$ \\ 1 Institute for Biodiversity and Ecosystem Dynamics, University of Amsterdam, \\ P.O. Box 94084, 1090 GB, Amsterdam, The Netherlands. \\ 2 Adaptive Dynamics Network, International Institute for Applied Systems Analysis, \\ Schlossplatz 1, 2361 Laxenburg, Austria. \\ * e-mail: egas@science.uva.nl \\ ^ e-mail: sabelis@science.uva.nl \\ $\S$ e-mail: dieckmann@iiasa.ac.at
}

Corresponding author: Martijn Egas, IBED - Population Biology, P.O.Box 94084, 1090 GB Amsterdam, The Netherlands; Street address: Kruislaan 320, 1098 SM, Amsterdam, The Netherlands. Fax: ++3120 5257754; e-mail: egas@science.uva.nl

LRH: MARTIJN EGAS ET AL.

RRH: SPECIALIZATION AND CHARACTER DISPLACEMENT.

Manuscript information: 7 keywords, 195 words in abstract, 5897 words in text, 6 Figures, 4 Appendixes and 3 Appendix Figures.

Resubmission manuscript for Evolution, 6 October 2004 


\begin{abstract}
We study the combined evolutionary dynamics of herbivore specialization and ecological character displacement, taking into account foraging behavior of the herbivores, and a quality gradient of plant types. Herbivores can adapt by changing two adaptive traits: their level of specialization in feeding efficiency and their point of maximum feeding efficiency along the plant gradient. The number of herbivore phenotypes, their levels of specialization, and the amount of character displacement among them are the result of the evolutionary dynamics, which is driven by the underlying population dynamics, which in turn is driven by the underlying foraging behavior. Our analysis demonstrates broad conditions for the diversification of a herbivore population into many specialized phenotypes, for basically any foraging behavior focusing utilization on highest gains while also including errors. Our model predicts two characteristic phases in the adaptation of herbivore phenotypes: a fast 'character displacement' phase, and a slow 'coevolutionary niche shift' phase. This two-phase pattern is expected to be of wide relevance in various consumer-resource systems. Bringing together ecological character displacement and the evolution of specialization in a single model, our study suggests that the foraging behavior of herbivorous arthropods is a key factor promoting specialist radiation.
\end{abstract}

Keywords: adaptive dynamics, herbivorous arthropods, resource gradient, ecological character displacement, coevolutionary niche shift, foraging behavior, adaptive radiation. 
Most herbivorous arthropods exploit one or a few plant species (Jaenike 1990; Schoonhoven et al. 1998). Why such specialists dominate is a major question (Berenbaum 1996). It is generally assumed that herbivores face a trade-off in utilizing different host plants. Thus, a specialist gains fitness on one host plant at the expense of fitness on others, whereas a generalist is a 'jack of all trades, but master of none' (Levins 1962, 1968; MacArthur and Levins 1964; Lawlor and Maynard Smith 1976; Rosenzweig 1981, 1987; Brown and Pavlovic 1992). Specialization is traditionally predicted to evolve under a strong trade-off, i.e., when density-independent fitness of a specialist on its resource is higher than that of a generalist on all resources combined (Levins 1962, 1968). However, when optimal foraging (or adaptive habitat choice) is included, specialization is even selected for under a weak trade-off (i.e., when a specialist gains less than it loses on other hosts), because inferior resources are avoided (Rosenzweig 1981, 1987; Wilson and Yoshimura 1994; Egas et al. 2004).

The models cited above did not explicitly consider the evolutionary dynamics of specialization. Adaptive dynamics theory provides tools for studying such processes in their proper frequency-dependent context (Metz et al. 1992, 1996a; Dieckmann and Law 1996; Dieckmann 1997; Geritz et al. 1998), by explicitly allowing ecological feedback into the evolutionary process (Heino et al. 1998). Evolutionary models of this type have highlighted how directional selection may drive a population towards a regime of disruptive selection, promoting adaptive radiation and speciation (Doebeli 1996; Geritz et al. 1998; Dieckmann and Doebeli 1999; Doebeli and Dieckmann 2000, 2003; Geritz and Kisdi 2000; Dieckmann et al. 2004; see also 
Rosenzweig 1978; Christiansen 1991; Brown and Pavlovic 1992; Abrams et al. 1993).

In this article, we extend previous evolutionary models of herbivore specialization by enhancing them with a combination of two key features. First, we include a continuum of plant types, which can be arranged along a (non-spatial) quality gradient, instead of considering only two discrete resources. Second, we allow errors in herbivore foraging that inevitably result, e.g., from sampling the environment or from sensory constraints (Stephens and Krebs 1986). Both extensions are inspired by what is known about the ecology of insect herbivores. Plants may well exhibit gradients in concentration of toxic or nutritive compounds, and insect herbivores are certainly not omniscient, nor do they move cost-free or redistribute instantaneously (Kennedy and Gray 1993). Thus, central assumptions underlying existing models of specialization are generally violated in nature (see, e.g., Ward 1987, 1992; Mayhew 1997; Grünbaum 1998; Schoonhoven et al. 1998).

These extensions enable an analysis that addresses the intricate coupling between (relatively realistic renditions of) three key biological processes, operating at different time scales (Fig. 1): evolutionary dynamics (slow pace; with our model accounting for both frequency- and density-dependent selection), population dynamics (intermediate pace; accounting for a full gradient of plant types), and behavioral dynamics (fast pace; accounting for inevitable errors in foraging activity).

By including a gradient of plant resources, we link the evolution of specialization to ecological character displacement, i.e., to the process by which natural selection on competing species in a community (or phenotypes in a population) leads to increased differences in the distribution of a quantitative character along a resource 
axis (Slatkin 1980). Three aspects, mostly neglected by the many preceding studies on character displacement, are addressed in this article.

First, most earlier studies investigated character displacement using ecological or quantitative genetic models with a fixed number of species (e.g., MacArthur and Levins 1967; Roughgarden 1972; May and MacArthur 1972; Slatkin 1980; Case 1981; Taper and Case 1985; for a review, see Taper and Case 1992b). Rummel and Roughgarden (1983, 1985), and Taper and Case (1992a,b) extended this approach by investigating whether there is room for an additional species at the coevolutionary equilibrium of a fixed number of species. These studies allowed invasion of a new species by migration from outside the coevolved system to increase the number of species (the fact that Rummel and Roughgarden's model allows for interspecific but not intraspecific frequency-dependent selection casts doubt on some of their results (see review in Taper and Case 1992b). Brown and Vincent (1992) studied ESS states of a predator-prey model, explicitly allowing for multiple species, without investigating, however, the selection pressures that would favor an increased number of species. In contrast, our model allows the emergence of new phenotypes when there is selection for a phenotype to split in two (i.e., when a phenotype comes under disruptive selection; migration will not be considered in our model). Accordingly, the number of species/phenotypes in our model is an emergent property of the population dynamical feedback into the evolutionary process.

Second, most earlier models of character displacement studied evolution of the distance between niches along a resource gradient, but limited attention to settings in which the niche width of species was kept constant (e.g., Slatkin 1980; see the review in Taper and Case 1992b). We are aware of only one exception: Taper and Case (1985) analyzed a more realistic quantitative genetic model where specializa- 
tion (called "within-phenotype niche width" in their article) could evolve as well.

This latter study, however, did not address the question whether increased specialization may allow invasion of additional species.

Third, and most importantly, earlier models of character displacement did not take foraging behavior into account. To our knowledge, the exceptions are Brew (1982, 1984), who studied character displacement in a purely ecological setting, with a fixed number of species and without the evolution of specialization. Therefore, the study presented here would appear to be the first to investigate the effects of foraging behavior on the combined evolution of character displacement and resource specialization. In our model, the number of herbivore phenotypes, their level of specialization, and the amount of character displacement among them are dynamically determined by the outcome of herbivore evolution, which in turn is driven by a reasonably realistic feedback involving the underlying population dynamics and behavioral dynamics (Fig. 1).

\section{MODEL DESCRIPTION}

In this section, we use standard descriptions of consumer-resource dynamics to formulate a model that is widely applicable to herbivore-plant systems, and particularly to herbivorous arthropods and their host plants. In the DISCUSSION we reflect on how alternative model assumptions may affect the results.

\section{Plant quality}

We consider plant types arranged along a quality gradient. The word "quality" is used here in a very specific sense: it reflects the ultimate potential for per capita growth of the herbivore. This notion is inspired by the observation from insect-plant 
biology that plants harbor intrinsic differences that are relevant for herbivores, irrespective of how well the arthropod in question is adapted to one plant species or the other. Such differences affect both the intrinsic growth rate and the carrying capacity of herbivores.

To highlight the relevance of such measures of quality in nature, we provide two examples. First, assume that plant quality is primarily determined by one limiting nutrient. Nitrogen content, for instance, has been recognized for years as a critical factor limiting herbivore growth (Slansky and Feeny 1977; McNeill and Southwood 1978; Mattson 1980; Denno and McClure 1983; Strong et al. 1984; White 1993; Fagan et al. 2002). Then, plant types can be ranked according to nitrogen content as a relevant measure of quality. Second, assume that plant quality is primarily determined by a measure of toxin content. The distribution of major secondary metabolites that function in plant defense is almost mutually exclusive among plant families (e.g., Wink 2003). Thus, plant types within one family produce similar secondary metabolites, and can be placed along a continuum from low to high quality. Of course, the relevant measure of plant quality can also be a combination of toxin and nutrient content. Correlations between such measures arise since plants with higher nutrient content are subject to selection for increased defense (Herms and Mattson 1992).

\section{Plant Population Dynamics}

In our model, the density $p(q)$ of plants with quality $q$ follows standard LotkaVolterra dynamics, 


$$
\frac{d}{d t} p(q)=r(q) \cdot p(q) \cdot\left[1-\frac{p(q)}{K(q)}\right]-c_{f} \cdot h \cdot u(q) \cdot p(q)
$$

Here, $r(q)$ and $K(q)$ are, respectively, the intrinsic growth rate and carrying capacity of plants with quality $q ; c_{f}$ is the feeding rate per plant per herbivore; $h$ is the number of herbivores; and $u$ is the herbivores' resource utilization spectrum, describing their proportional utilization of plant qualities (see Foraging Behavior). The dynamics in eq. 1 thus describe changes in the herbivores' resource availability spectrum $p(q)$.

\section{Feeding Efficiency}

Feeding efficiency describes how well a herbivore can digest a specific plant quality. Since the quality of plants is determined by a diversity of toxins and/or nutrients, and since feeding efficiency depends on a large number of underlying physiological traits, high feeding efficiency on toxins and/or nutrients of a plant with one quality does not necessarily imply high feeding efficiency on the - different - toxins and/or nutrients of a plant with another quality. Accordingly, herbivores face a trade-off in feeding efficiency when exploiting different plant qualities. They can either specialize by becoming highly efficient on relatively narrow intervals of the plant quality gradient or forego such high efficiency and instead adjust their physiology in a generalist manner to achieve intermediate feeding efficiency across all plant qualities. This trade-off is modeled by assuming a normal distribution of feeding efficiency along the plant quality gradient,

$$
e(q)=\exp \left[-\frac{1}{2}(\mu-q)^{2} / \sigma^{2}\right] /(\sqrt{2 \pi} \sigma) .
$$


The mean $\mu$ of the efficiency spectrum $e(q)$ determines the focal range of plant qualities utilized by a herbivore, while the standard deviation $\sigma$ defines the herbivore's niche width and thus determines its level of specialization (see Kassen 2002 for a review of empirical data). A low value of $\sigma$ implies a relatively high efficiency in a narrow range of the plant quality gradient, whereas a high value results in a relatively low efficiency, yet in a wide range. We study the joint evolution of the adaptive traits $\mu$ and $\sigma$.

\section{Foraging Behavior}

We assume that behavior underlying habitat choice is driven by maximization of food intake, and that changes in foraging behavior occur on a fast time scale relative to population dynamics. Optimally foraging herbivores will redistribute as soon as their feeding has decreased the density of the currently most attractive plant quality to an extent that plants of different quality yield more. Therefore, the distribution of herbivores over the plant gradient can change continuously over time (Krivan 1997). The gain $g(q)$ that an individual herbivore can extract from plants of quality $q$ is defined as its intake rate when feeding on those plants exclusively. This is a product of plant quality $q$, feeding rate $c_{f}$, plant biomass $p(q)$, and feeding efficiency $e(q)$ (which depends on $\mu$ and $\sigma$, as described in eq. 2),

$$
g(q)=q \cdot c_{f} \cdot p(q) \cdot e(q)
$$

This gain function $g(q)$ describes a herbivore's intake rate from feeding on plants of quality $q$ and determines its per capita growth rate, as will be specified in detail be- 
low (see Herbivore Population Dynamics). Therefore, $g(q)$ is an appropriate measure for herbivores to decide which plants to feed on.

Foraging behavior is generally subject to error, preventing individuals from foraging optimally. Such errors may result from various causes, such as the need of individuals to sample their environment before being able to make a decision, or from constraints on the sensory perception and mobility of foragers. In our model, sub-optimal levels of foraging are described phenomenologically by means of a flexible power law, so that the utilization $u(q)$ of plants with quality $q$ is proportional to $g(q)^{\alpha}$,

$$
u(q)=C(\sigma) \frac{g(q)^{\alpha}}{\int g(q)^{\alpha} d q}
$$

The denominator above is only needed to ensure proper normalization of the probability distribution $u$. The factor $C(\sigma)$ reflects the fact that specialists need more time to search for the specific plants they are specialized on and therefore can spend less time exploiting those plants. We describe this phenomenologically by $C(\sigma)=\sigma /(\sigma+$ $c_{s}$ ), where the half-saturation constant $c_{s}$ measures the cost of specialization.

The parameter $\alpha$ can take any value in the range $[0,+\infty)$ and provides a conveniently flexible way of describing a herbivore's foraging accuracy. Setting $\alpha$ to zero yields non-selective feeding (utilization is uniform and completely independent of gain), whereas letting $\alpha$ go to infinity yields dynamic optimal foraging (utilization is strictly limited to resources offering maximal gain). Values of $\alpha$ in between these two extremes result in selective, but sub-optimal foraging (Fig. 2). For example, when $\alpha=1$, the utilization $u(q)$ of plants with quality $q$ is proportional to the gain 
$g(q)$ from those plants. We do not consider evolutionary change in $\alpha$, since foraging errors act as evolutionary constraints that naturally result from sampling the environment and from limitations in sensory perception and mobility.

\section{Herbivore Population Dynamics}

The per capita birth and death rates of herbivores are functions of their intake rate, $I$. The latter is the product of gain and utilization, integrated over the gradient of plant qualities,

$$
I=\int g(q) \cdot u(q) d q
$$

Individuals can produce offspring if their intake rate exceeds a threshold $I_{\min }$. Above this threshold, the birth rate $b(I)$ is a saturating function of intake (Fig. 3). The death rate $d(I)$ is asymptotically declining from the starvation mortality $d_{\max }$ towards a background mortality $d_{\min }$ (Fig. 3). In the model, we used the functions $b(I)=b_{\max }\left(1-\exp \left[c_{b}\left(I_{\min }-I\right)\right]\right)_{+}$and $d(I)=d_{\min }+\left(d_{\max }-d_{\min }\right) \exp \left[-c_{d} \cdot I\right]$. Here, $b_{\text {max }}$ is the maximum birth rate, and $c_{b}$ is a scaling constant determining the slope of increase. The function $(\ldots)_{+}$maps any negative values to zero. For the mortality rate, $c_{d}$ is a scaling constant determining the slope of decrease. A herbivore's per capita growth rate is $b(I)-d(I)$, so that the number of herbivores, $h$, changes according to

$$
\frac{d}{d t} h=[b(I)-d(I)] \cdot h
$$

Note that the intake rate $\hat{I}$ at population dynamical equilibrium satisfies $b(\hat{I})=d(\hat{I})$. 


\section{Evolutionary Dynamics}

The herbivore's fitness landscape is determined by the invasion fitness of rare phenotypes, defined as the long-term per capita growth rate of such phenotypes in the environment determined by the resident population at its population dynamical equilibrium. The expected rate of deterministic phenotypic evolution is then proportional to the local fitness gradient. An individual-based version of the model is used to investigate higher degrees of polymorphism; this also allows checking the robustness of conclusions obtained from the deterministic model. (See Appendix I for a full description.)

\section{RESULTS}

We first examine two extreme cases: non-selective foraging and optimal foraging, the latter of which is shown to yield unrealistic evolutionary outcomes. Because optimal foraging is also biologically unrealistic, the remainder of this section focuses on sub-optimal foraging.

\section{Non-selective Foraging: $\alpha=0$}

In general, the equilibrium of the plant-herbivore population dynamics cannot be derived analytically, and this therefore also applies to the invasion fitness of herbivores. However, for non-selective foraging, we can show that all equilibrium plant densities $\hat{p}(q)$ are negatively correlated with $\hat{h}$, the equilibrium number of herbivores (see Appendix II). A mutant herbivore that can invade a population of resident herbivores, must decrease the plant densities to lower levels than $\hat{p}(q)$ in order to grow, causing the resident type to decline in density. Consequently, the mu- 
tant can invade the resident population if and only if it decreases plant densities to lower levels than $\hat{p}(q)$, and thereby grows to a higher equilibrium $\hat{h}$. This implies that an optimization principle (Mylius and Diekmann 1995; Metz et al. 1996b) exists under non-selective foraging: herbivore evolution then leads to a strategy $(\mu, \sigma)$ that maximizes the equilibrium number of herbivores by minimizing the equilibrium density of plants. An optimal strategy in such a scenario possesses three important features (Meszéna et al. 2001): it is evolutionarily singular (selection pressures vanish at the optimal strategy), it is convergence stable (gradual evolution converges on the optimal strategy), and it is evolutionarily stable (no other strategy can invade the evolving population once the optimal strategy has been attained). Such a strategy is referred to as a continuously stable strategy (CSS; Eshel 1983).

Which exploitation strategy will be optimal under non-selective foraging? Notice that under non-selective foraging plant densities at population dynamical equilibrium are proportional to their carrying capacities, making the herbivore's intake $\hat{I}$ is proportional to $C(\sigma) \int q \cdot K(q) \cdot e(q) d q$ (see Appendix II). This function is maximized when the mean $\mu$ of $e(q)$ is at the maximum of $q \cdot K(q)$. The standard deviation $\sigma$ of $e(q)$ for which intake is maximized depends on the specialization $\operatorname{cost} c_{s}: \sigma$ increases from zero when $c_{s}$ increases from zero. Simulations tracking the evolutionary dynamics in the strategy $(\mu, \sigma)$ confirm these results. Under non-selective foraging, therefore, it always pays to specialize on the plant quality offering the best compromise between quality $q$ and abundance $K$, even though non-selective herbivores cannot avoid using the entire gradient of plant qualities. 


\section{Optimal Foraging: $\alpha \rightarrow \infty$}

To understand the population dynamics under optimal foraging, consider the situation in which all plant types are at their carrying capacity and a herbivore phenotype with strategy $(\mu, \sigma)$ and enters the system. (We assume throughout this section that optimal foragers cannot have a foraging cost of specialization, $c_{s}=0$.) Typically, a single plant quality will offer the highest gain to this herbivore. The herbivores will start depleting that plant quality (and, because of optimal foraging, only that quality), thereby decreasing its gain. At some point, adjacent plant qualities will yield gains equal to the initially exploited one, and thus the herbivores will start using the corresponding range of plant qualities uniformly. This process will continue until the herbivores reach their population dynamical equilibrium. Here, the gain distribution over the quality gradient will resemble a 'table mountain'. All plant qualities used will yield equal gain (given by $\hat{I}$; see Appendix III), and the herbivores' utilization spectrum will be rectangular, implying equal utilization of all plant qualities used. Plant qualities with a gain below $\hat{I}$ are not used at all by the optimally foraging herbivores, and therefore their equilibrium densities remain at carrying capacity.

Again, the population dynamical equilibrium and thus the resultant invasion fitness cannot be derived analytically. We can conclude, however, that under optimal foraging no evolutionarily singular strategy exists: any monomorphic population of resident herbivores can always be invaded by any mutant of small effect, since such mutants have a higher efficiency for at least one specific plant quality that is in use by the resident population. These plant qualities then yield a gain higher than $\hat{I}$ (because they yield a gain equal to $\hat{I}$ for the resident individuals), and, as the new phenotype forages optimally, it starts using these plant qualities exclusively and thus attains a positive growth rate (see Appendix III). 
For polymorphic herbivore populations an analogous line of reasoning applies: any new herbivore phenotype can invade if its efficiency function yields a gain higher than $\hat{I}$ on any of the plant qualities. This conclusion changes as soon as all plant qualities are utilized by the herbivores. Then, herbivores with efficiency functions broadened relative to that of residents cannot invade any more, since they find the plant quality for which their gain is highest exploited to a level lower than $\hat{I}$. By contrast, more specialized herbivores can always invade a more generalist 'nichesharer' (i.e., a phenotype with a larger value of $\sigma$, but the same value of $\mu$; see Appendix III). Therefore, evolution under optimal foraging leads to a theoretically infinite diversity of extreme specialists (each with a population density approaching zero) at all plant qualities $q$ at which plant density is high enough to sustain such specialists.

\section{Sub-optimal Foraging - Deterministic Approximation}

Herbivore evolution under sub-optimal foraging $(0<\alpha<\infty)$ is analyzed numerically. For the sake of brevity, we describe the evolutionary dynamics for $\alpha=1$ (i.e., with utilization proportional to gain) and $c_{s}=0$ (i.e., without a cost of specialization). Appendix IV shows that this case is representative for a wide range of $\alpha$ values and for specialization costs that are not too high.

Starting out with a single herbivore strategy, gradual evolution of herbivore phenotypes leads to a globally attractive singular strategy (Fig. 4a), where the herbivores homogeneously utilize almost the entire resource gradient (Fig. 4b), creating a gain curve much like the 'table mountain' of optimal foragers. This singular strategy is an evolutionary branching point: when the fitness gradient for both $\mu$ and $\sigma$ has declined to zero, mutants of small effect can still invade the resident population (in 
contrast to the case of a CSS; Geritz et al. 1998). The resultant disruptive selection can cause the population to become dimorphic.

During such evolutionary branching, one herbivore phenotype initially specializes on higher-quality plants while the other becomes more generalist and focuses on lower-quality plants (Fig. 5a). This divergence arises because low-quality plants are underexploited at the branching point. Therefore, herbivores can do better either by specializing on higher-quality plants or by becoming generalists on lower-quality plants.

While the two phenotypes diverge in trait space, their mutual impact through the feedback on plant densities declines. To quantify this, two characteristics are useful: the intersection point $q^{*}$ of the utilization spectra of the two phenotypes, and their total overlap $\Delta u$. The intersection point $q^{*}$ describes the plant quality along the resource axis at which the two phenotypes exhibit equal utilization; it can be interpreted as the border between their foraging ranges. By contrast, the total utilization overlap $\Delta u$ (i.e., the roughly triangular areas in Figs. 5d-f, peaked at $q^{*}$ ) measures the amount of competition between the two phenotypes. Plotting $q^{*}$ and $\Delta u$ against each other reveals two distinct phases in the coevolutionary process (Fig. 5b).

In the first, 'character displacement' phase, competition drives the two phenotypes apart: the total utilization overlap quickly declines, while the intersection point shifts towards higher qualities. This is a fast process, indicating large fitness benefits for avoiding competition. The two phenotypes evolve strategies that lead them to forage on distinctly different plant quality ranges (compare Figs. 5d and 5e). During this phase, the lower-quality phenotype increases in abundance by exploiting an increasingly wider range of the plant gradient, while the number of individuals in the higher-quality phenotype declines by specializing on a small but high-quality range 
of the plant gradient (Fig. 5c). At the end of this phase, total utilization overlap is minimized (Fig. 5e), and with it the strength of competition between the two phenotypes (see Fig. 5b).

In the second, 'coevolutionary niche shift' phase, the higher-quality phenotype still experiences selection for specialization: generalists cannot invade into its population, as the resident phenotype already uses the entire range of resources between the high-quality border of the plant gradient and the range dominated by the lowerquality phenotype. This reduces the realized niche width of the higher-quality phenotype, which, in turn, gives evolutionary opportunity for the lower-quality phenotype to expand its niche width. Hence, the intersection point progresses slowly but steadily towards higher plant qualities, whereas the total utilization overlap essentially remains constant (Fig. 5b). This process causes the evolutionary trajectory of the lower-quality phenotype to change direction (Fig. 5a).

The coevolutionary process ends up at a pair of strategies, with the lower-quality phenotype being more generalized and the higher-quality type more specialized (Fig. 5a). Their utilization spectra come close to the rectangular shapes of optimal foragers (Fig. 5f). These two singular strategies are again evolutionary branching points, so that subsequent evolutionary change can lead to trimorphic and quadrimorphic herbivore populations.

\section{Sub-optimal Foraging - Individual-based Model}

Analyzing the continued evolutionary dynamics becomes tedious using the deterministic approximation - using an individual-based model is then more efficient (see Appendix I for details). Evolution in the individual-based model resembles adaptive radiation in the $\mu$-dimension, leading to many specialized types (Fig. 6). However, in 
contrast to the deterministic approach, the 'coevolutionary niche shift' phase is generally absent: typically, a new split already occurs before this phase sets in. This is because mutational steps are finite and reproduction is asexual in the individualbased model. While weak directional selection would still be expected to drive the 'coevolutionary niche shift' phase, herbivores are already so close to the fitness minimum that even small mutational steps suffice to jump across this fitness minimum, thus initiating a split that would not yet have occurred if mutational steps were infinitely small or if speciation is slow. Specifically, a sexual population is expected to linger much longer at a fitness minimum before, if ever, disruptive selection can cause reproductive isolation. Sexual herbivores must thus be expected to display both coevolutionary phases as characterized above.

The resulting community of specialists eventually becomes impervious to further invasion by any herbivore phenotype. On the one hand, more generalist herbivores, even though they can use a wider range of the gradient, are outcompeted everywhere by the specialized herbivores already present. On the other hand, more specialized herbivores have to pay a larger cost of specialization, causing them to lose the competition against the resident specialists. Because of this global evolutionary stability, it does not matter how the evolutionary 'game' is started. To confirm this, we considered a variation of our model in which $1 \%$ of the mutants are chosen at random from the full range of phenotypes, instead of resembling their parents. Widely different herbivore phenotypes are then quickly established; yet the resultant evolutionary outcome is indistinguishable from a community built up through gradual evolution by small mutational steps.

Not surprisingly, the number of specialists in the resulting community decreases when the cost of specialization is increased. However, the degree of ecological char- 
acter displacement among the specialists is not affected by this cost (see Appendix IV, Fig. A3).

\section{DISCUSSION}

We have shown that, along a gradient of plant quality, frequency-dependent selection emerging from competition under sub-optimal foraging can lead to the build-up of a community of increasingly specialized herbivore phenotypes (Figs. 5, 6). Such diversification (reminiscent of adaptive radiation; Schluter 2000a,b) follows a characteristic evolutionary pattern and occurs for a wide range of specialization costs and of foraging accuracies. Because of this broad robustness, our findings are likely to be applicable to a variety of consumers other than herbivores.

\section{Alternative Assumptions}

To what extent do the results reported here depend on underlying assumptions? For reasons of tractability, our individual-based model neglects sexual reproduction. By contrast, the deterministic approximation of evolutionary dynamics, in which the rate of evolutionary change is proportional to the local fitness gradient, applies to sexual models (Lande 1979) just as well as to asexual models (Dieckmann and Law 1996). In general, a distinction between sexual and asexual evolution is not critical as long as genetics are additive and mutations do not have too large an effect (e.g., Taylor 1989; Charlesworth 1990; Christiansen 1991; Iwasa et al. 1991; Taper and Case 1992a; Abrams et al. 1993a; Weissing 1996; Van Dooren 2004), and frequencydependent selection does not favor evolutionary branching (e.g., Dieckmann and Doebeli 1999; Abrams 2001; Van Dooren 2004). It must be kept in mind, however, that recombination promoting sufficient cohesion of a sexual population's gene pool 
(e.g., Felsenstein 1981; Dieckmann and Doebeli 1999) is expected to delay or even prevent some of the branching events (see Fig. 6). Interestingly, such delays underscore the importance of the two characteristic coevolutionary phases identified in this study (see below).

A second set of critical assumptions relates to preventing unstable population dynamics by assuming a linear functional response and logistic plant growth. Alternative assumptions resulting in equally stable population dynamics (e.g., saturating functional response and semi-chemostat plant growth) will not affect the results qualitatively. If, however, the potential for unstable dynamics is incorporated, increasing herbivore specialization may lead to population dynamical destabilization. The resulting degree of specialization may then be lower, because generalists can cope better with resource fluctuations (Slatkin and Lande 1976; Rosenzweig 1987; Brown 1989; Wilson and Yoshimura 1994). This particular ecological feedback into the evolutionary process of specialization deserves future research.

Finally, the assumption of a linear trade-off in feeding efficiency $e(q)$ does not appear to be critical. Nonlinear trade-offs (implying that $\int e(q) d q$ decreases or increases with $\sigma$, resulting in so-called weak and strong trade-offs, respectively) can be viewed as imposing an alternative cost of specialization/generalization. Strong tradeoffs are expected to lead to even more specialization, but weak trade-offs might favor generalists (and therefore prevent evolutionary branching), especially when foraging is not or only weakly density-dependent. However, simulations (not shown here) indicate that weaker trade-offs do not generally seem to select for generalists, even under conditions of very sub-optimal foraging $(\alpha<1)$. This makes sense, because the intake rate of herbivores is unaffected when the cost of specialization is applied to efficiency $e$ (affecting gain $g$ ) instead of utilization $u$ (see eq. 5). 
The Interplay between Ecological Character Displacement and Specialization By assuming a gradient of resources, we studied the joint evolution of the focal point as well as the range of resource utilization. In this way, we explicitly analyzed the joint evolution of specialization and ecological character displacement. Most earlier models of character displacement studied evolution of distance between niches along a resource gradient, but kept the niche width constant (see the review by Taper and Case (1992b); see also Taper and Case (1985) for a model in which specialization, called "within-phenotype niche width" in their model, could evolve as well). Interestingly, evolution of specialization and ecological character displacement have not often been brought into contact before, possibly because character displacement studies usually deal with evolution of the range of resources used, and not with evolution of the efficiency of resource utilization.

Key insights from our model, therefore, arise from the interplay between the evolutionary processes of specialization and character displacement. First, the primary evolutionary branching in the herbivore population always results in a specialist utilizing heavily exploited plants of high quality and a generalist utilizing plant qualities that are less extensively used. Whenever the underlying resource gradient is asymmetric, the likelihood of the initial divergence being completely neutral in $\sigma$ is negligible. In any such consumer-resource model, combining character displacement with specialization evolution, we must therefore expect the displacement to result in one consumer species initially becoming more generalist. Clearly, at short evolutionary time scales Simpson's (1953; Schluter 2000a) empirical view that specialists descend from generalist ancestors in an adaptive radiation does not hold. However, 
given a long enough evolutionary time scale, our model is in agreement with Simpson's observation (Fig. 6).

A second important aspect of the interplay between specialization and character displacement is the prediction of two characteristic phases in the divergence of a species pair: a fast 'character displacement' phase, during which overlap in the plant utilization of herbivore species is minimized, is typically followed by a slow 'coevolutionary niche shift' phase, during which herbivore species jointly adjust their niches leading to maximized overall plant utilization. Also this two-phase pattern of consumer coevolution is likely to apply to other consumer-resource systems.

\section{Limiting Similarity and the Evolution of Specialization}

We have shown that optimal foraging $(\alpha \rightarrow \infty)$ in our model would lead to an unrealistic, infinite diversity of extreme specialists, each exploiting an infinitely narrow part of the plant gradient (reminiscent of a conjecture by Vincent et al. 1993 for Lotka-Volterra models in which the resource gradient was unbounded and the competitive effects among species were smaller than those within species). In this case, limiting similarity between the phenotypes would approach zero, and species packing along the plant gradient would be infinitely dense. In their original study on limiting similarity, MacArthur and Levins (1967) proved that rectangular utilization curves allow for infinite species packing, but a biological interpretation was not given. Here we have shown that these curves apply to consumers capable of dynamical optimal foraging.

Optimal foraging sensu stricto is a biologically unrealistic assumption, because it requires omniscience and unlimited mobility. Real foragers need to sample their environment: herbivorous arthropods need time to select their host plant, exhibit stage- 
dependent mobility, and mothers may not always select the best host for their (immobile) offspring (e.g., Wainhouse and Howell 1983; Rauscher 1983; Rauscher and Papaj 1983; Whitham 1983; Robertson 1987; Moran and Whitham 1990; Valladares and Lawton 1991; Underwood 1994). Evidence is accumulating that they can approximate optimal foraging by adaptive learning (Dukas and Bernays 2000; Egas and Sabelis 2001; Egas et al. 2003; Nomikou et al. 2003). Our model incorporates such behavior as sub-optimal foraging.

We have shown that sub-optimal foraging $(0<\alpha<\infty)$ limits the number of specialist herbivores evolving. Due to sub-optimal foraging, the range of plant qualities a herbivore species exploits always exceeds the range on which it is specialized, thereby leaving less opportunity for other specialists to invade. Consequently, the number of specialist herbivores decreases with the foraging accuracy $\alpha$ (Appendix IV). Adding a cost of specialization to the foraging behavior also limits the number of phenotypes, by limiting their levels of specialization (Appendix IV). In line with our findings, Mitchell (2000) showed a similar limit to species richness along a gradient as a consequence of increasing travel costs. He also noted the possibility of evolutionary branching (using the term 'evolutionarily stable minima').

Non-selective foraging of the herbivores $(\alpha=0)$ in our model leads to a single continuously stable strategy (CSS), instead of a polymorphism. Therefore, limiting similarity does not apply. The herbivore CSS is, in terms of the physiological definition we use in this paper, a specialist strategy, even though it cannot help but utilize the entire gradient of plant qualities (and hence remains a generalist when defined in terms of foraging behavior). 


\section{Ecological Character Displacement}

The degree of ecological character displacement in the evolutionarily stable community depends on the level of sub-optimal foraging, but not on the cost of specialization (Appendix IV). The better a herbivore is able to home in on plants with higher gains, the better it can keep its realized niche separate from its competitors. Consequently, the degree of ecological character displacement, based on the evolved fundamental niche characteristics ( $\mu$ and $\sigma$ ), will be lower for more discriminate foragers. The cost of specialization does not influence character displacement because it does not affect the ability of herbivores to minimize competition with other herbivores by foraging on disjunct ranges of the plant gradient.

An important factor affecting ecological character displacement in previously studied models is the shape of the carrying capacity distribution (MacArthur and Levins 1967; Slatkin 1980; Taper and Case 1985; Drossel and McKane 1999, 2000). Character displacement is favored when this distribution is uniform (not dropping off towards the edges, as a Gaussian distribution does). For such uniform distributions, phenotypes diverging in trait space incur no fitness cost that may outweigh the benefits of reduced competition. Because we took foraging behavior into account, we need to consider the shape of the gain curve $g$ rather than that of the carrying capacity $K$. Any selective foraging behavior flattens the gain curve, and hence favors character displacement - irrespective of the shape of $K$.

A fundamental open question is whether a single 'quality' axis can approximately describe how herbivorous arthropods experience plant types. In general, the growth rate of herbivorous insects appears to be limited by nutrients, rather than by energy (Schoonhoven et al. 1998: chapter 4). Usually, one axis for proteins and one for carbohydrates will capture most variation in growth rate (Simpson and Raubenheimer 
1996; Raubenheimer and Simpson 1999). However, other axes may be important, e.g., due to the presence of secondary plant metabolites (increasing toxicity or decreasing digestibility). The general effects of increasing the dimension of trait spaces on the properties of evolutionary branching points are still largely unknown. Yet, we propose that adding more quality dimensions will increase, rather than decrease, the possibilities for frequency-dependent selection to induce adaptive radiation (see also Yoshiyama and Roughgarden 1977; Pacala and Roughgarden 1982), simply because a multiplicity of trait dimensions provides more opportunity for specialization and ecological character displacement.

\section{ACKNOWLEDGEMENTS}

We thank André Noest, Mikko Heino, Steph Menken, Tad Kawecki, Sergey Gavrilets, Franjo Weissing, Jonathan Losos, and five anonymous reviewers for comments on this work. ME gratefully acknowledges funding by the Netherlands Organization for Scientific Research NWO for participation in IIASA's Young Scientists Summer Program. UD gratefully acknowledges financial support by the Austrian Science Fund; by the Austrian Federal Ministry of Education, Science, and Cultural Affairs; and by the European Research Training Network ModLife (Modern Life-History Theory and its Application to the Management of Natural Resources), funded through the Human Potential Programme of the European Commission. 


\section{LITERATURE CITED}

Abrams, P.A. 2001. Modelling the adaptive dynamics of traits involved in inter- and intraspecific interactions: An assessment of three methods. Ecol. Lett. 4: 166-175.

_ genetic and ESS models. Evolution 47: 982-985.

— H. Matsuda, and Y. Harada. 1993b. Evolutionarily unstable fitness maxima and stable fitness minima of continuous traits. Evol. Ecol. 7: 465-487.

Berenbaum, M.R. 1996. Introduction to the symposium: on the evolution of specialization. Am. Nat. 148: S78-S83.

Brew, J.S. 1982. Niche shift and the minimisation of competition. Theor. Popul. Biol. $22: 367-381$.

-1984. An alternative to Lotka-Volterra competition in coarse-grained environments. Theor. Popul. Biol. 25: 265-288.

Brown, J.S. 1989. Coexistence on a seasonal resource. Am. Nat. 133: 168-182.

—, and N.B. Pavlovic. 1992. Evolution in heterogeneous environments: effects of migration on habitat specialization. Evol. Ecol. 6: 360-382.

— evolutionary game. Evolution 46: 1269-1283.

Case, T.J. 1981. Niche packing and coevolution in competition communities. PNAS 78: 5021-5025.

Charlesworth, B. 1990. Optimization models, quantitative genetics, and mutation. Evolution 44: 520-538.

Christiansen, F.B. 1991. On the conditions for evolutionary stability for a continuously varying character. Am. Nat. 138: 37-50.

Denno, R. F., and M. S. McClure. 1983. Variable plants and herbivores in natural and managed systems. Academic Press, New York.

Dieckmann, U. 1997. Can adaptive dynamics invade? Trends Ecol. Evol 12: 128131.

— , and M. Doebeli. 1999. On the origin of species by sympatric speciation. Nature 400: 354-357.

—, and R. Law. 1996. The dynamical theory of coevolution: A derivation from stochastic ecological processes. J. Math. Biol. 34: 579-612.

—, M. Doebeli, J.A.J. Metz, and D. Tautz. 2004. Adaptive Speciation. Cambridge Univ. Press, Cambridge, UK. 
Doebeli, M. 1996. A quantitative genetic competition model for sympatric speciation. J. Evol. Biol. 9: 893-909.

Doebeli, M., and U. Dieckmann. 2000. Evolutionary branching and sympatric speciation caused by different types of ecological interactions. Am. Nat. 156: S77S101.

- 2003. Speciation along environmental gradients. Nature 421: 259-264.

Drossel, B., and A. McKane. 1999. Ecological character displacement in quantitative genetic models. J. Theor. Biol. 196: 363-376.

. 2000. Competitive speciation in quantitative genetic models. J. Theor. Biol. 204: 467-478.

Dukas, R., and E.A. Bernays. 2000. Learning improves growth rate in grasshoppers. PNAS 97: 2637-2640.

Egas, M., and M.W. Sabelis. 2001. Adaptive learning of host preference in a herbivorous arthropod. Ecol. Lett. 4: 190-195.

—, U. Dieckmann, and M.W. Sabelis. 2004. Evolution restricts the coexistence of specialists and generalists: the role of trade-off structure. Am. Nat. 163: 518531.

— D.-J. Norde, and M.W. Sabelis. 2003. Adaptive learning in arthropods: spider mites learn to distinguish food quality. Exp. Appl. Acarol. 30: 233-247.

Eshel, I. 1983. Evolutionary and continuous stability. J. Theor. Biol. 103: 99-111.

Fagan, W.F., E. Siemann, C. Mitter, R.F. Denno, A.F. Huberty, H.A. Woods, and J.J. Elser. 2002. Nitrogen in insects: implications for trophic complexity and species diversification. Am. Nat. 160: 784-802.

Felsenstein, J. 1981. Skepticism towards Santa Rosalia, or why are there so few kinds of animals? Evolution 35: 124-138.

Geritz, S.A.H., and É. Kisdi. 2000. Adaptive dynamics in diploid, sexual populations and the evolution of reproductive isolation. Proc. Roy. Soc. B 267: 1671-1678.

—, É. Kisdi, G. Meszéna, and J.A.J. Metz. 1998. Evolutionary singular strategies and the adaptive growth and branching of the evolutionary tree. Evol. Ecol. 12: $35-57$.

Grünbaum, D. 1998. Using spatially explicit models to characterize foraging performance in heterogeneous landscapes. Am. Nat. 151: 97-115.

Heino, M., J.A.J. Metz, and V. Kaitala. 1998. The enigma of frequency-dependent selection. Trends Ecol. Evol 13: 367-370. 
Herms, D., and W. Mattson. 1992. The dilemma of plants: to grow or defend. Q. Rev. Biol. 67: 283-335.

Iwasa, Y., A. Pomiankowski, and S. Nee. 1991. The evolution of costly mate preferences. II. The "handicap" principle. Evolution 45: 1431-1442.

Jaenike, J. 1990. Host specialization in phytophagous insects. Annu. Rev. Ecol. Syst. 21: $243-273$.

Kassen, R. 2002. The experimental evolution of specialists, generalists, and the maintenance of diversity. J. Evol. Biol. 15: 173-190.

Kennedy, M., and R.D. Gray. 1993. Can ecological theory predict the distribution of foraging animals - a critical analysis of experiments on the ideal free distribution. Oikos 68: 158-166.

Krivan, V. 1997. Dynamic ideal free distribution: effects of optimal patch choice on predator-prey dynamics. Am. Nat. 149: 164-178.

Lande, R. 1979. Quantitative genetic analysis of multivariate evolution, applied to brain:body size allometry. Evolution 33: 402-416.

Lawlor, L.R., and J. Maynard Smith. 1976. The coevolution and stability of competing species. Am. Nat. 110: 79-99.

Levins, R. 1962. Theory of fitness in a heterogeneous environment. I. The fitness set and adaptive function. Am. Nat. 96: 361-373.

- 1968. Evolution in changing environments. Princeton Univ. Press, Princeton.

MacArthur, R.H., and R. Levins. 1964. Competition, habitat selection and character displacement in a patchy environment. PNAS 51: 1207-1210.

- 1967. The limiting similarity, convergence and divergence of coexisting species. Am. Nat. 101: 377-385.

Mattson, W. J. 1980. Herbivory in relation to plant nitrogen content. Annu. Rev. Ecol. Syst. 11: 119-161.

May, R.M., and R.H. MacArthur. 1972. Niche overlap as a function of environmental variability. PNAS 69: 1109-1113.

Mayhew, P.J. 1997. Adaptive patterns of host-plant selection by phytophagous insects. Oikos 79: 417-428.

McNeill, S., and T. R. E. Southwood. 1978. The role of nitrogen in the development of insect/plant relationships. Pages 77-98 in J. S. Harborne, ed. Aspects of plant and animal coevolution. Academic Press, London. 
Meszéna G., É. Kisdi, U. Dieckmann, S.A.H. Geritz, and J.A.J Metz 2001. Evolutionary optimisation models and matrix games in the unified perspective of adaptive dynamics. Selection 2: 193-210.

Metz, J.A.J., Nisbet, R.M., and Geritz, S.A.H. 1992. How should we define "fitness" for general ecological scenarios? Trends Ecol. Evol. 7: 198-202.

— S.A.H. Geritz, G. Meszéna, F.J.A. Jacobs, and J.S. van Heerwaarden, 1996a. Adaptive dynamics, a geometrical study of the consequences of nearly faithful reproduction. Pp. 183-231 in Van Strien, S.J., and S.M. Verduyn Lunel, eds. Stochastic and Spatial Structures of Dynamical Systems. North Holland, Amsterdam, The Netherlands.

— S.D. Mylius, and O. Diekmann. 1996b. When does evolution optimize? on the relation between types of density dependence and evolutionarily stable life history parameters. IIASA Working Paper WP-96-04, available at http://www.iiasa. ac.at/Research/ADN/Series.html.

Mitchell, W.A. 2000. Limits to species richness in a continuum of habitat heterogeneity: an ESS approach. Evol. Ecol. Res. 2: 293-316.

Moran, N.A., and T.G. Whitham. 1990. Differential colonization of resistant and susceptible host plants: Pemphigus and Populus. Ecology 71: 1059-1067.

Mylius, S.D., and O. Diekmann. 1995. On evolutionarily stable life histories, optimization and the need to be specific about density dependence. Oikos 74: 218-224.

Nomikou, M., A. Janssen, and M.W. Sabelis. 2003. Herbivore host plant selection: whitefly learns to avoid host plants that are unsafe for her offspring. Oecologia 136: 484-488.

Pacala, S.W., and J. Roughgarden. 1982. The evolution of resource partitioning in a multidimensional resource space. Theor. Popul. Biol. 22: 127-145.

Raubenheimer, D., and S.J. Simpson. 1999. Integrating nutrition: a geometrical approach. Entomol. Exp. Appl. 91: 67-82.

Rauscher, M.D. 1983. Alteration of oviposition behavior by Battus philenor butterflies in response to variation in host-plant density. Ecology 64: 1028-1034.

— , and D.R. Papaj. 1983. Demographic consequences of discrimination among conspecific host plants by Battus philenor butterflies. Ecology 64: 1402-1410.

Robertson, H.G. 1987. Oviposition site selection in Cactoblastis cactorum (Lepidoptera): constraints and compromises. Oecologia 73: 601-608.

Rosenzweig, M.L. 1978. Competitive speciation. Biol. J. Linn. Soc. 10: 275-289. 
- 1981. A theory of habitat selection. Ecology 62: 327-335.

1987. Habitat selection as a source of biological diversity. Evol. Ecol. 1:

315-330.

Roughgarden, J. 1972. Evolution of niche width. Am. Nat. 106: 683-718.

Rummel, J.D., and J. Roughgarden. 1983. Some differences between invasionstructured and coevolution-structured competitive communities: a preliminary theoretical analysis. Oikos 41: 477-486.

.1985. A theory of faunal buildup for competition communities. Evolution 39: 1009-1033.

Schluter, D. 2000a. The Ecology of Adaptive Radiation. Oxford Univ. Press, Oxford.

-2000b. Ecological character displacement in adaptive radiation. Am. Nat. 156: S4-S16.

Schoonhoven, L.M., T. Jermy, and J.J.A. van Loon. 1998. Insect-Plant Biology: From physiology to evolution. Chapman and Hall, London.

Simpson, G.G. 1953. The major features of evolution. Columbia Univ. Press, New York.

Simpson, S.J., and D. Raubenheimer. 1996. Feeding behaviour, sensory physiology and nutritional feedback: a unifying model. Entomol. Exp. Appl. 80: 55-64.

Slansky, F., and P. Feeny. 1977. Stabilization of the rate of nitrogen accumulation by larvae of the cabbage butterfly on wild and cultivated plants. Ecol. Monogr. 47: $209-228$.

Slatkin, M. 1980. Ecological character displacement. Ecology 61: 163-177.

— independent model. Am. Nat. 110: 31-55.

Stephens, D.W., and J.R. Krebs. 1986. Foraging Theory. Princeton University Press, Princeton.

Strong, D. R., J. H. Lawton, and T. R. E. Southwood. 1984. Insects on plants. Harvard University Press, Cambridge, Mass.

Taper, M.L., and T.J. Case. 1985. Quantitative genetic models for the coevolution of character displacement. Ecology 66: 355-371.

. 1992a. Models of character displacement and the theoretical robustness of taxon cycles. Evolution 46: 317-333.

. 1992b. Coevolution among competitors. Oxford Surveys in Evolutionary Biology 8: 63-109. 
Taylor, P.D. 1989. Evolutionary stability in one-parameter models under weak selection. Theor. Popul. Biol. 36: 125-143.

Tilman, D. 1982. Resource competition and community structure. Princeton Univ. Press, Princeton.

Underwood, D.L.A. 1994. Intraspecific variability in host plant quality and ovipositional preferences in Eucheira socialis (Lepidoptera: Pieridae). Ecol. Entomol. 19: 245-256.

Valladares, G., and J.H. Lawton. 1991. Host-plant selection in the holly leaf-miner: does mother know best? J. Anim. Ecol. 60: 227-240.

Van Dooren, T.J.M. 2004. Adaptive dynamics for Mendelian genetics. In press in Dieckmann, U., and J.A.J. Metz, eds. Elements of Adaptive Dynamics. Cambridge Univ. Press, Cambridge.

Vincent, T.L., Y. Cohen, and J.S. Brown. 1993. Evolution via strategy dynamics. Theor. Popul. Biol. 44: 149-176.

Wainhouse, D., and R.S. Howell. 1983. Intraspecific variation in beech scale populations and in susceptibility of their host Fagus sylvatica. Ecol. Entomol. 8: 351-359.

Ward, S.A. 1987. Optimal habitat selection in time-limited dispersers. Am. Nat. 129: 568-579.

- 1992. Assessing functional explanations of host-specificity. Am. Nat. 139: 883-891.

Weissing, F.J. 1996. Genetic versus phenotypic models of selection: can genetics be neglected in a long-term perspective? J. Math. Biol. 34: 533-555.

White, T. C. R. 1993. The inadequate environment: nitrogen and the abundance of animals. Springer, New York.

Whitham, T.G. 1983. Host manipulation of parasites: within plant variation as a defense against rapidly evolving pests. Pp. 15-41 in Denno, R.F., and M.S. McClure eds. Variable Plants and Herbivores in Natural and Managed Systems. Academic Press, New York, USA.

Wilson, D.S., and J. Yoshimura. 1994. On the coexistence of specialists and generalists. Am. Nat. 144: 692-707.

Wink, M. 2003. Evolution of secondary metabolites from an ecological and molecular phylogenetic perspective. Phytochemistry 64: 3-19.

Yoshiyama, R.M, and J. Roughgarden. 1977. Species packing in two dimensions. Am. Nat. 111: 107-121. 


\section{APPENDIX I — ADAPTIVE DYNAMICS}

The theory of adaptive dynamics describes evolutionary processes in asexual populations under frequency-dependent selection, explicitly incorporating the inherently stochastic nature of these processes, and offering techniques for simplifying their analysis (Metz et al. 1992, 1996; Dieckmann 1994; Dieckmann and Law 1996; Dieckmann 1997; Geritz et al. 1997, 1998). Individual-based models, including both demographic and mutational stochasticity, are the natural starting point for such a framework. Such models, however, are very time-consuming and often cumbersome and inconclusive to study. Therefore, in addition to using individual-based models, we base our investigation on a deterministic approximation, the canonical equation of adaptive dynamics. In this systematically derived approximation, the dynamics of resident populations are treated deterministically and the evolutionary change that arises from small mutational steps expressed by successful invaders deterministically follows the local gradient of the fitness landscape around a resident phenotype. Assuming that in long-term asexual evolution relevant mutations occur at low probability (mutation-limited evolution), adaptation can be studied by evaluating the fate of mutants in populations that consist of one or more resident phenotypes at their ecological equilibrium (here we use the term 'ecological equilibrium' to describe the joint outcome of behavioral dynamics and population dynamics).

More specifically, the fitness landscape of adaptive dynamics is determined by the invasion fitness of mutant phenotypes, defined as the long-term per capita growth rate of a rare mutant in the environment set by the resident population at its ecological equilibrium (Metz et al. 1992). A resident phenotype can be invaded and ousted by mutant phenotypes with positive invasion fitness. Accounting for the demo- 
graphic stochasticity of such advantageous mutants, the resultant rate of phenotypic change is proportional to the local fitness gradient (Dieckmann and Law 1996), in line with analogous findings for models based on quantitative genetics (Lande 1979; see also Abrams 2001).

When the adaptive dynamics eventually reach a phenotype at which the fitness gradient vanishes, it has attained a so-called evolutionarily singular strategy. Singular strategies can be of several types (Dieckmann 1997; Geritz et al. 1997, 1998; see also Christiansen 1991), two of which are encountered in this study: continuously stable strategies and evolutionary branching points. A continuously stable strategy (CSS; Eshel 1983) is a strategy that is an evolutionary attractor (i.e., a singular strategy that is convergence stable) and that also cannot be invaded by any neighboring phenotype (i.e., the singular strategy is locally evolutionarily stable). Note that an ESS differs from a CSS in that the former does not require the first condition to be satisfied (i.e., an ESS is not necessarily an evolutionary attractor; Eshel and Motro 1981). By contrast, an evolutionary branching point is an evolutionary attractor that is not evolutionarily stable (Metz et al. 1992, 1996; Geritz et al. 1998). Mutants close to such a singular strategy have a chance to mutually invade each other and form a stable dimorphism. The resulting dimorphic adaptive dynamics allow for two resident phenotypes and describe the initial phenotypic divergence and subsequent evolutionary change in the two subpopulations. Such a pair is again expected to converge to a singular strategy, the evolutionary stability of which can once more be determined by evaluating whether mutants of small effect around the singular strategy are able to invade.

Whereas the deterministic approximation is a versatile tool for investigating the adaptive dynamics under low degrees of polymorphism (monomorphic and dimor- 
phic evolution) it becomes a bit tedious thereafter. An individual-based model, by contrast, is naturally suited to investigating adaptive dynamics involving multiple evolutionary branching events. Therefore we use an individual-based version of the deterministic model to investigate higher degrees of polymorphism. At the same time this model allows checking the robustness of evolutionary conclusions obtained from the deterministic approximation when relaxing the simplifying assumptions that approximation is based on (e.g., mutation-limited evolution and small mutational steps).

In the individual-based model, all individual herbivores can be assigned different phenotypes $(\mu, \sigma)$, and demography as well as mutations are treated stochastically. Based on herbivore birth and death rates from the deterministic equations above, the waiting times for the next birth or death event to take place are drawn from an exponential probability distribution (Gillespie 1976; Van Kampen 1981; Dieckmann 1994; Dieckmann et al. 1995). During a birth event, the phenotype of offspring individuals can either be faithfully inherited from their parent, or it can be affected by mutation. The latter occurs with probability $p_{m}$, and the mutant phenotype is then drawn from a normal distribution with standard deviation $s_{m}$ around the parental phenotype.

\section{APPENDIX II — NON-SELECTIVE FORAGING: $\alpha=0$}

Under non-selective foraging, herbivore utilization is uniformly distributed over the plant quality gradient: the utilization spectrum $u(q)$ is flat. Since total utilization must equal $C(\sigma)$ (see eq. 4), the utilization spectrum is then given by 


$$
u(q)=\frac{C(\sigma)}{q_{\max }-q_{\min }} .
$$

Using this, we can solve for the equilibrium plant densities,

$$
\frac{d}{d t} p(q)=0=r(q) \cdot \hat{p}(q) \cdot\left[1-\frac{\hat{p}(q)}{K(q)}-\frac{c_{f} \cdot \hat{h} \cdot C(\sigma)}{r(q) \cdot\left(q_{\max }-q_{\min }\right)}\right],
$$

which gives

$$
\hat{p}(q)=0 \quad \text { or } \quad \hat{p}(q)=K(q) \cdot\left[1-\frac{c_{f} \cdot \hat{h} \cdot C(\sigma)}{\left(q_{\max }-q_{\min }\right) \cdot r(q)}\right] \text {. }
$$

The second solution shows that (provided a positive equilibrium is attained) $\hat{p}(q)$ is negatively related to the equilibrium number of herbivores. Note also that $\hat{p}(q)$ is proportional to $K(q)$, owing to non-selective foraging. In turn, the equilibrium number of herbivores can be expressed by using the equation for $\hat{p}(q)$ in the equilibrium intake rate $\hat{I}$,

$$
\begin{aligned}
\hat{I} & =\frac{c_{f} \cdot C(\sigma)}{q_{\max }-q_{\min }} \int_{q_{\min }}^{q_{\max }} \hat{p}(q) \cdot e(q) \cdot q d q \\
& =\frac{c_{f} \cdot C(\sigma)}{q_{\max }-q_{\min }} \int_{q_{\min }}^{q_{\max }} e(q) \cdot q \cdot K(q) \cdot\left[1-\frac{c_{f} \cdot \hat{h} \cdot C(\sigma)}{\left(q_{\max }-q_{\min }\right) \cdot r(q)}\right] d q .
\end{aligned}
$$

By splitting up the integral and solving for $\hat{h}$ we obtain 


$$
\hat{h}=\frac{\int_{q_{\min }}^{q_{\max }} e(q) \cdot q \cdot K(q) d q-\frac{\left(q_{\max }-q_{\min }\right) \cdot \hat{I}}{c_{f} \cdot C(\sigma)}}{\int_{q_{\min }} e(q) \cdot q \cdot K(q) \frac{c_{f} \cdot C(\sigma)}{\left(q_{\max }-q_{\min }\right) \cdot r(q)} d q} .
$$

These equations cannot be solved explicitly. Therefore, also the invasion fitness of herbivores cannot be determined analytically. Nevertheless, conditions for a mutant to invade a resident population at equilibrium can be established, using the criterion that for invasion to succeed the intake rate of a mutant, $I^{\prime}$, must be larger than the equilibrium intake rate $\hat{I}$ (which is the same for mutant and resident, and is here for convenience expressed using the mutant's strategy),

$$
I^{\prime}=\frac{c_{f} \cdot C\left(\sigma^{\prime}\right)}{q_{\max }-q_{\min }} \int_{q_{\min }}^{q_{\max }} \hat{p}(q) \cdot e^{\prime}(q) \cdot q d q>\hat{I}=\frac{c_{f} \cdot C\left(\sigma^{\prime}\right)}{q_{\max }-q_{\min }} \int_{q_{\min }}^{q_{\max }} \hat{p}^{\prime}(q) \cdot e^{\prime}(q) \cdot q d q
$$

Here, $e^{\prime}(q)$ is the efficiency function of the mutant and $\hat{p}^{\prime}(q)$ is the equilibrium plant density function for a population of such mutants. This results in the inequality

$$
\int_{q_{\min }}^{q_{\max }} \hat{p}(q) \cdot e^{\prime}(q) \cdot q d q>\int_{q_{\min }}^{q_{\max }} \hat{p}^{\prime}(q) \cdot e^{\prime}(q) \cdot q d q
$$

This invasion condition is satisfied when the equilibrium plant densities over all plant qualities are smaller in a population of mutant-type herbivores than in a population of resident-type herbivores (i.e., if $\hat{p}^{\prime}(q)<\hat{p}(q)$ for all $q$ between $q_{\text {min }}$ and $\left.q_{\text {max }}\right)$. This is the case when the mutant strategy leads to a higher equilibrium herbi- 
vore abundance, since it is shown above that equilibrium plant densities are negatively related to equilibrium herbivore abundance. Therefore, a mutant can invade a resident population if and only if its strategy leads to a higher equilibrium herbivore abundance (or, equivalently, to lower equilibrium plant densities).

\section{APPENDIX III - OPTIMAL FORAGING: $\alpha \rightarrow \infty$}

At ecological equilibrium, a monomorphic population of herbivores uses the range of plant qualities that form the plateau top of the 'table mountain'-shaped gain curve, between $q_{\min }$ and $q_{\max }$ (this description and the analysis below readily carry over to situations where the gain distribution dictates a disjunct utilization distribution for the optimal foragers). Therefore, for $q<q_{\min }$ and $q>q_{\max }$, the utilization density vanishes, $u(q)=0$, and hence the equilibrium plant densities are at their carrying capacities, $\hat{p}(q)=K(q)$. By contrast, for $q_{\min } \leq q \leq q_{\max }$ the utilization spectrum is constant and given by

$$
u(q)=\frac{1}{q_{\max }-q_{\min }} .
$$

(We assume no cost of specialization, $c_{s}=0$, which means that $C(\sigma)=1$.) As follows from the assumption of optimal foraging, the gain $g(q)$ is also constant over the utilized range of plant qualities, denoted by $g_{\max }$. Thus, we can solve for the equilibrium intake rate $\hat{I}$,

$$
\hat{I}=\int_{q_{\min }}^{q_{\max }} g(q) \cdot u(q) d q=\frac{g_{\max }}{q_{\max }-q_{\min }} \cdot\left(q_{\max }-q_{\min }\right)=g_{\max } .
$$


This equation shows that, at equilibrium, the maximum gain $g_{\max }$ equals $\hat{I}$.

As in Appendix II, the equilibrium density of herbivores and plants cannot be solved explicitly. Therefore, to investigate the possibility for mutants to invade, we again have to resort to studying the intake rate. In between $q_{\min }$ and $q_{\max }$, the gain of a mutant $g^{\prime}(q)$ for a specific plant quality $q$ is larger than that of the resident when

$$
g^{\prime}(q)=q \cdot c_{f} \cdot \hat{p}(q) \cdot e^{\prime}(q)>\hat{I}=q \cdot c_{f} \cdot \hat{p}(q) \cdot e(q)
$$

which holds for any plant quality for which $e^{\prime}(q)>e(q)$. Due to the optimal foraging assumption, the mutant can restrict feeding to the plant quality that yields highest gain. This will result in an intake rate higher than $\hat{I}$ when the above inequality holds, allowing such a mutant to invade. For arbitrarily small mutations in either $\mu$ or $\sigma$, and given that $q_{\max }-q_{\min }$ is not arbitrarily small, this inequality holds: efficiency is higher for plant qualities in the neighborhood of $q=\mu$. When the inequality does not hold, also the plant quality ranges that the resident population does not use $\left(q<q_{\text {min }}\right.$ and $\left.q>q_{\max }\right)$ should be investigated to check whether at any plant quality the gain exceeds the equilibrium intake rate,

$$
K(q) \cdot e^{\prime}(q) \cdot q>\hat{I}
$$

If the whole quality gradient is in use by resident herbivore types, only the first of the above inequalities applies. To be certain that a mutant $\left(\mu, \sigma^{\prime}\right)$ can invade, we have to consider its gain at plant quality $q=\mu$. As shown above, this gain exceeds $\hat{I}$ 
if $e^{\prime}(\mu)>e(\mu)$, which always applies when $\sigma^{\prime}<\sigma$ (i.e., for a more specialized mu$\operatorname{tant})$.

$$
\text { APPENDIX IV - SUB-OPTIMAL FORAGING: } 0<\alpha<\infty
$$

Deterministic approximation — Tracking the singular point of a monomorphic population through trait space for different values of the cost of specialization $c_{s}$ shows that a higher cost of specialization indeed selects for more generalist phenotypes (Fig. A1). For increasing values of $\alpha$, the singular strategy is less specialized for low $c_{s}$ and more specialized for extremely high $c_{s}\left(c_{s}>3\right.$; see Fig. A1). Whether the singular strategy is an evolutionary branching point also depends on the cost of specialization (Fig. A2). Below a critical cost, evolutionary branching results in specialized herbivore phenotypes. Above the critical cost, the evolutionary dynamics result in a single generalist phenotype. This critical cost is higher if $\alpha$ is higher, i.e., if the herbivores are better foragers.

Individual-based model — At the polymorphic evolutionary equilibrium, the number of phenotypes in the herbivore community depends on the degree of sub-optimal foraging, $\alpha$ : higher values of $\alpha$ allow for more phenotypes to coexist in the population (Fig. A3a). This is because more selective foragers can better focus their foraging effort, even when they are specialized. At the same time, the phenotypes are less specialized when they are more selective foragers (Fig. A3b). Increasing the cost of specialization decreases the number of phenotypes that can coexist along the plant gradient (Fig. A3a). The number of phenotypes that 'squeeze into' the resource gradient goes down (limiting similarity), because the herbivores evolve a lower degree of specialization when the cost for specialization is higher (Fig. A3b). 
The degree of ecological character displacement can be expressed by the so-called $d / w$ ratio (May and McArthur 1972). Here, $d$ is the distance between two adjacent phenotypes, measured by the means $\mu_{i}$ of their efficiency distributions, $d=\left|\mu_{1}-\mu_{2}\right|$. Through the $d / w$ ratio, the distance $d$ is then considered relative to the width $w$ of the efficiency distribution of the focal phenotype, which is here taken as the standard deviation $\sigma$ of the efficiency distribution. In this way, each comparison of a pair of adjacent phenotypes gives two values of the $d / w$ ratio (one for each phenotype) and the total number of $d / w$ ratios is $2 \cdot($ number of branches -1 ). The $d / w$ ratio describes displacement in the fundamental niches of the phenotypes, not in their realized niches (which are kept roughly separate, due to their foraging behavior). It turns out that this measure of character displacement remains fairly constant along the plant quality gradient. Values for $d / w$ increase from roughly 2 to 6 as $\alpha$ decreases from 1 to 0.1 , but are not significantly affected by the cost of specialization (Fig. A3c). The latter result is expected, since this cost does not affect foraging ability, so that the separation between phenotypes along the plant gradient (which is caused by this ability) remains unchanged. Thus, the amount of ecological character displacement of herbivore phenotypes depends critically on their degree of sub-optimal foraging behavior.

\section{LITERATURE CITED IN APPENDIXES}

Abrams, P.A. 2001. Modelling the adaptive dynamics of traits involved in inter- and intraspecific interactions: An assessment of three methods. Ecol. Lett. 4: 166-175.

Dieckmann U. 1994. Coevolutionary Dynamics of Stochastic Replicator Systems. Central Library of the Research Center Juelich, Juelich, Germany. 1997. Can adaptive dynamics invade? Trends Ecol. Evol 12: 128-131. 
, and R. Law. 1996. The dynamical theory of coevolution: A derivation from stochastic ecological processes. J. Math. Biol. 34: 579-612.

_ P. Marrow, and R. Law. 1995. Evolutionary cycling in predator-prey interactions: Population dynamics and the red queen. J. Theor. Biol. 178: 91-102.

Eshel, I. 1983. Evolutionary and continuous stability. J. Theor. Biol. 103: 99-111. , and U. Motro. 1981. Kin selection and strong evolutionary stability of mutual help. Theor. Popul. Biol. 19: 420-433.

Geritz S.A.H., J.A.J. Metz, É. Kisdi, and G. Meszéna .1997. Dynamics of adaptation and evolutionary branching. Phys. Rev. Lett. 78: 2024-2027.

—, É. Kisdi, G. Meszéna, and J.A.J. Metz. 1998. Evolutionary singular strategies and the adaptive growth and branching of the evolutionary tree. Evol. Ecol. 12: $35-57$.

Gillespie, D.T. 1976. A general method for numerically simulating the stochastic time evolution of coupled chemical reactions. J. Comp. Phys. 22: 403-434.

Lande, R. 1979. Quantitative genetic analysis of multivariate evolution, applied to brain:body size allometry. Evolution 33: 402-416.

May, R.M., and R.H. MacArthur. 1972. Niche overlap as a function of environmental variability. PNAS 69: 1109-1113.

Metz JAJ, R.M. Nisbet, and S.A.H. Geritz. 1992. How should we define "fitness" for general ecological scenarios? Trends Ecol. Evol 7: 198-202.

— , S.A.H. Geritz, G. Meszéna, F.J.A. Jacobs, and J.S. van Heerwaarden, 1996. Adaptive dynamics, a geometrical study of the consequences of nearly faithful reproduction. Pp. 183-231 in Van Strien, S.J., and S.M. Verduyn Lunel, eds.

Stochastic and Spatial Structures of Dynamical Systems. North Holland, Amsterdam, The Netherlands.

Van Kampen, N.G. 1981. Stochastic processes in physics and chemistry. North Holland, Amsterdam, The Netherlands. 


\section{FIGURE CAPTIONS}

Figure 1 Our model describes the interplay between processes at three different time scales. At the fast behavioral time scale, herbivores forage and adjust their utilization spectrum $u(q)$ along the gradient of plant qualities $q$. At the intermediate population dynamical time scale, plant densities $p(q)$ and herbivore abundances $h$ are changing. At the slow evolutionary time scale, the physiology of herbivores adapts, resulting in modifications of their efficiency spectrum $e(q)$. A feedback to the behavioral dynamics occurs since population dynamics and evolutionary dynamics affect the gain $g(q)$ that a herbivore can extract from plant quality $q$, leading to altered utilization.

Figure 2 Utilization spectra along the plant quality gradient, for a hypothetical gain function (proportional to the thick solid curve) and different values of the foraging accuracy parameter $\alpha$. The distribution for $\alpha=1$ is simply proportional to the gain distribution (thick continuous curve). The other distributions shown are for $\alpha=0$ (dashed curve; non-selective foraging: herbivores use all existing plant qualities with equal probability), $\alpha=0.1$ (dotted curve), $\alpha=2$ (thin continuous curve), and $\alpha \rightarrow \infty$ (vertical line with arrow; optimal foraging: herbivores use only the plant quality yielding the highest gain).

Figure 3 Birth and death rates as functions of intake rate. Herbivores can produce offspring if their intake rate exceeds a threshold $I_{m i n}$. Above this threshold, the birth rate $b(I)$ is a saturating function of intake. The death rate $d(I)$ is asymptotically declining from the starvation mortality $d_{\max }$ towards a background mortality $d_{\min }$. Growth rate is zero for an intake rate $\hat{I}$ that satisfies $b(\hat{I})=d(\hat{I})$. 
Figure 4 Monomorphic evolution of the herbivores' efficiency strategy $(\mu, \sigma)$ in a population with sub-optimal foraging. (a) Evolutionary phase portrait, showing two isoclines and the directions of evolutionary change in between. The continuous curve is the evolutionary isocline for the mean $\mu$, and the dashed curve is the isocline for the standard deviation $\sigma$. The evolutionarily singular strategy, where both isoclines cross, is an evolutionary branching point. (b) Utilization spectrum along the plant gradient at population dynamical equilibrium, as determined by the singular strategy in (a). Parameters: $K(q)=50+10 \cdot N_{0.1}(q-0.5), r(q)=1, b_{\max }=1.0, c_{f}=0.0025$, $c_{b}=400, c_{d}=1200, c_{S}=0, d_{\max }=1.0, d_{\min }=0.02, I_{\min }=0.0005$, and $\alpha=1.0$.

Figure 5 Dimorphic evolution of the herbivores' efficiency strategy. (a) Evolutionary trajectories of the two phenotypes. Starting from the primary evolutionary branching point (Fig. 4a), subsequent evolutionary change proceeds as indicated by the arrows. Positions 1, 2, and 3 in panels (a) to (c) correspond to the starting point, turning point, and end point of dimorphic evolution, respectively. Utilization spectra from positions 1, 2, and 3 are presented in panels (d)-(f), respectively. (b) Covariation of total utilization overlap between the two phenotypes $(\Delta u)$ and of the intersection point of their utilization spectra $\left(q^{*}\right)$. The first, fast phase of evolutionary change features ecological character displacement, strongly reducing the utilization overlap, whereas the second, slow phase is characterized by coevolutionary niche shift: the intersection point shifts to higher plant qualities, while the total utilization overlap remains essentially constant. (c) Resultant changes in herbivore abundances (thin curves; the herbivore morph specializing on low plant qualities has the higher densities). Total herbivore abundance is shown by the thick curve and increases until the end of the character displacement phase (position 2). (d) Initial utilization spectra of the two phenotypes in the population, corresponding to position 
1 in panels (a)-(c). (e) Utilization spectra of the two phenotypes at the end of the character displacement phase, corresponding to positions 2 in panels (a)-(c). (f) Utilization spectra of the two phenotypes at the dimorphic evolutionary attractor, corresponding to positions 3 in panels (a)-(c). Parameters as in Fig. 4.

Figure 6 Polymorphic evolution of the herbivore's efficiency strategy in the individual-based model. The left panel shows the evolution of a diversity of phenotypes along the plant quality gradient (black branches consist of more than 200 individuals, dark gray branches of 100-200 individuals, and light gray branches of less than 100 individuals). The right panel shows the concomitant changes in the standard deviation $\sigma$ of the herbivores' efficiency spectra. Specialization sharply increases as the number of branches increases. Parameters as in Fig. 4; except for: $s_{m}=0.005$, $p_{m}=0.0003, r(q)=3$, and $c_{S}=0.1$.

Figure A1 Evolutionarily singular herbivore strategies $(\mu, \sigma)$ for different foraging accuracies, $\alpha$, and costs of specialization, $c_{s}$. Filled circles: $\alpha=0.01$; open circles: $\alpha=0.1$; open diamonds: $\alpha=0.5$; filled triangles: $\alpha=1.0$; open squares: $\alpha=5.0$; open triangles: $\alpha=10$. Values of $c_{S}$ as indicated in the figure. Other parameters as in Fig. 4.

Figure A2 Critical values of the cost of specialization, $c_{S}$, for different foraging accuracies, $\alpha$. Above the curve, the evolutionarily singular strategy is a continuously stable: at evolutionary equilibrium the population consists of one herbivore phenotype with a generalist strategy. Below the curve, the singular strategy is an evolutionary branching point: here, the population can split up in two or more herbi- 
vore phenotypes with increasing degrees of specialization. Other parameters as in Fig. 4.

Figure A3 Characteristics of the polymorphic evolutionary equilibrium for different foraging accuracies, $\alpha$, and costs of specialization, $c_{s}$. (a) Number of phenotypes (open circles: $\alpha=0.1$; filled circles: $\alpha=0.5$; filled squares: $\alpha=1.0$ ). Note that for $\alpha=0.1$ evolutionary branching only occurs for $c_{s}=0$; therefore, in this case the number of phenotypes is 1 for $c_{s}=0.01$ and $c_{s}=0.1$. (b) Average level of specialization $\sigma$ among the phenotypes. (c) Average character displacement $d / w$ among the phenotypes. Other parameters as in Fig. 6. 
Figure 1

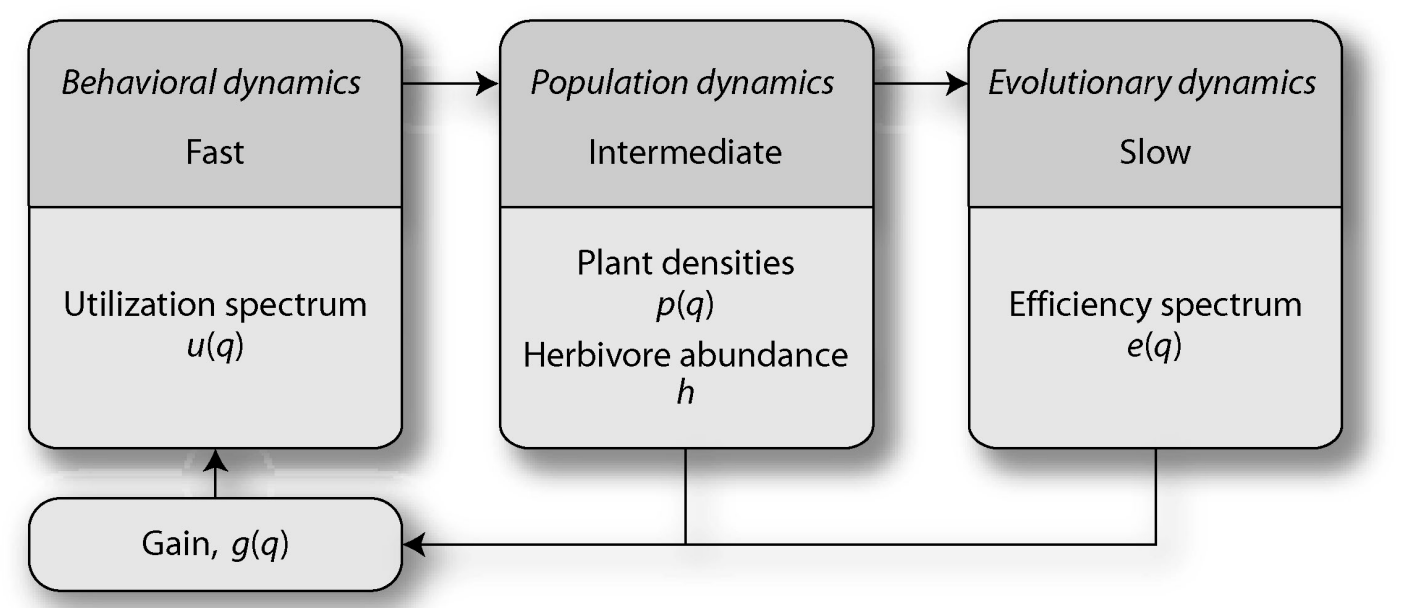


Figure 2

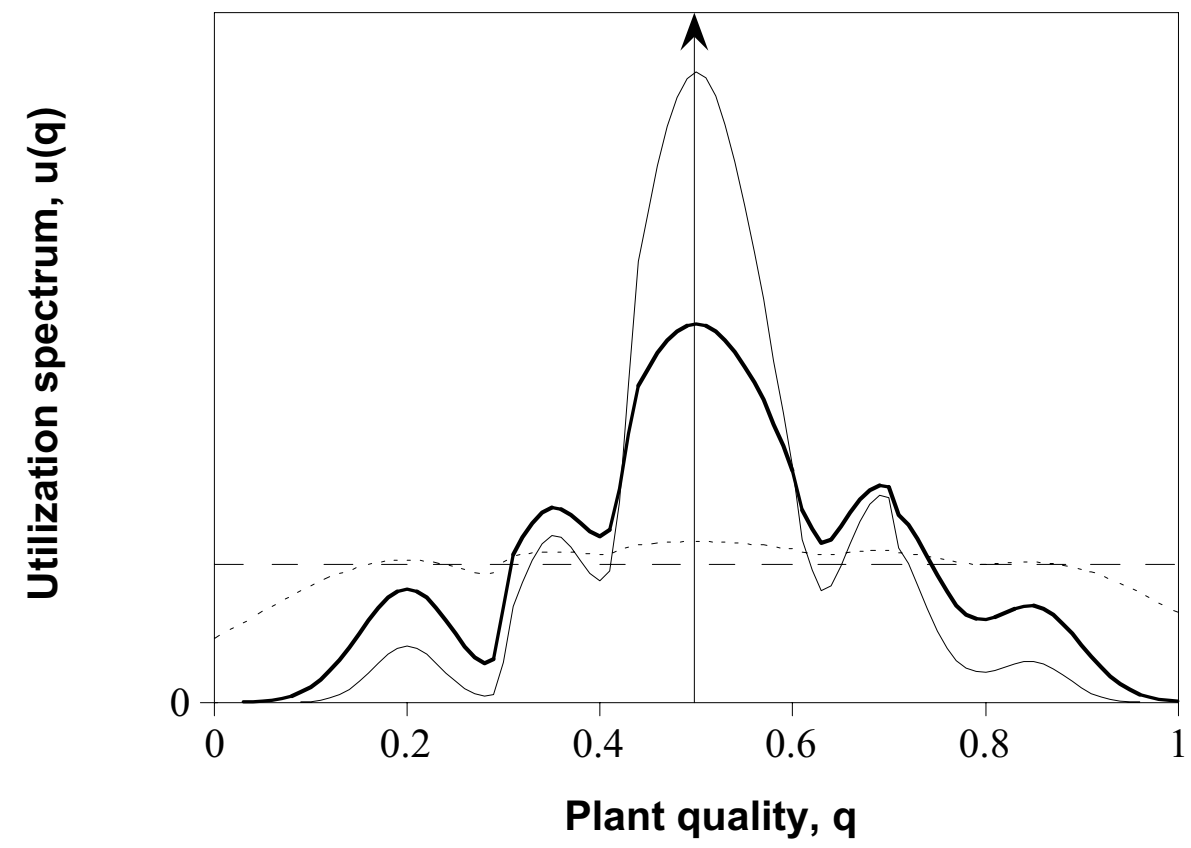


Figure 3

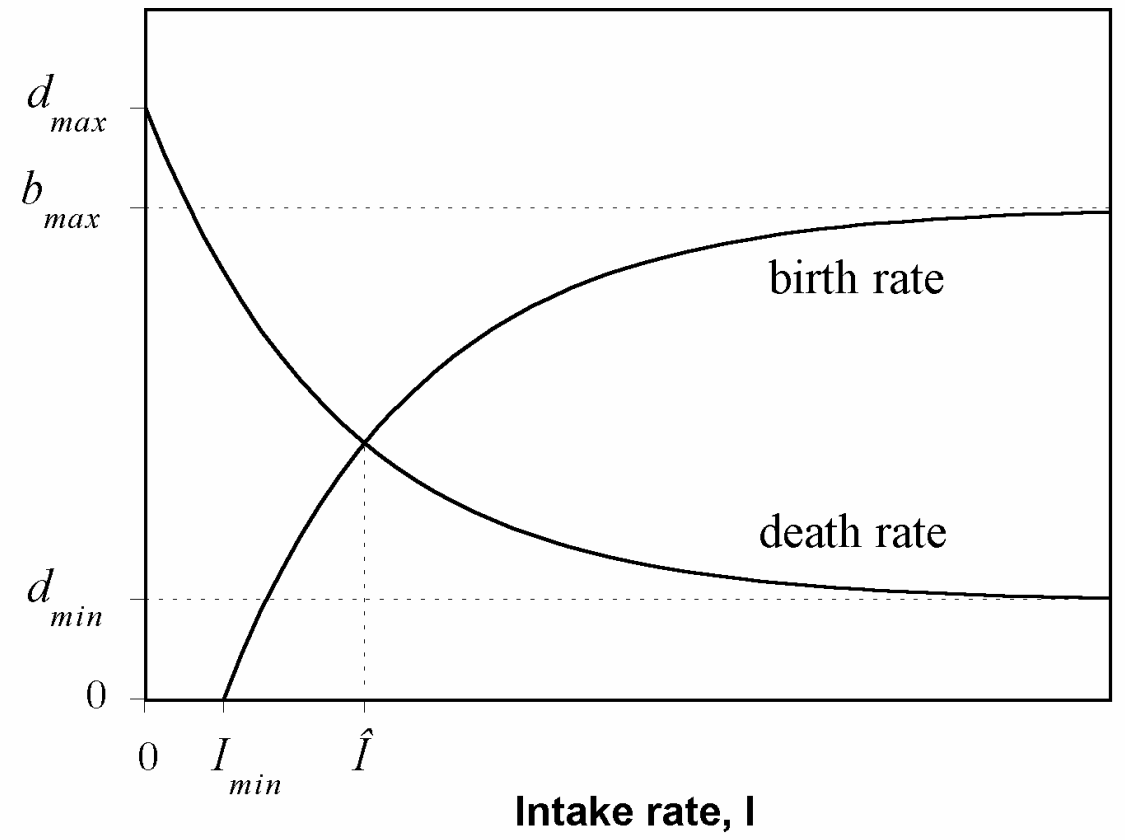


Figure 4
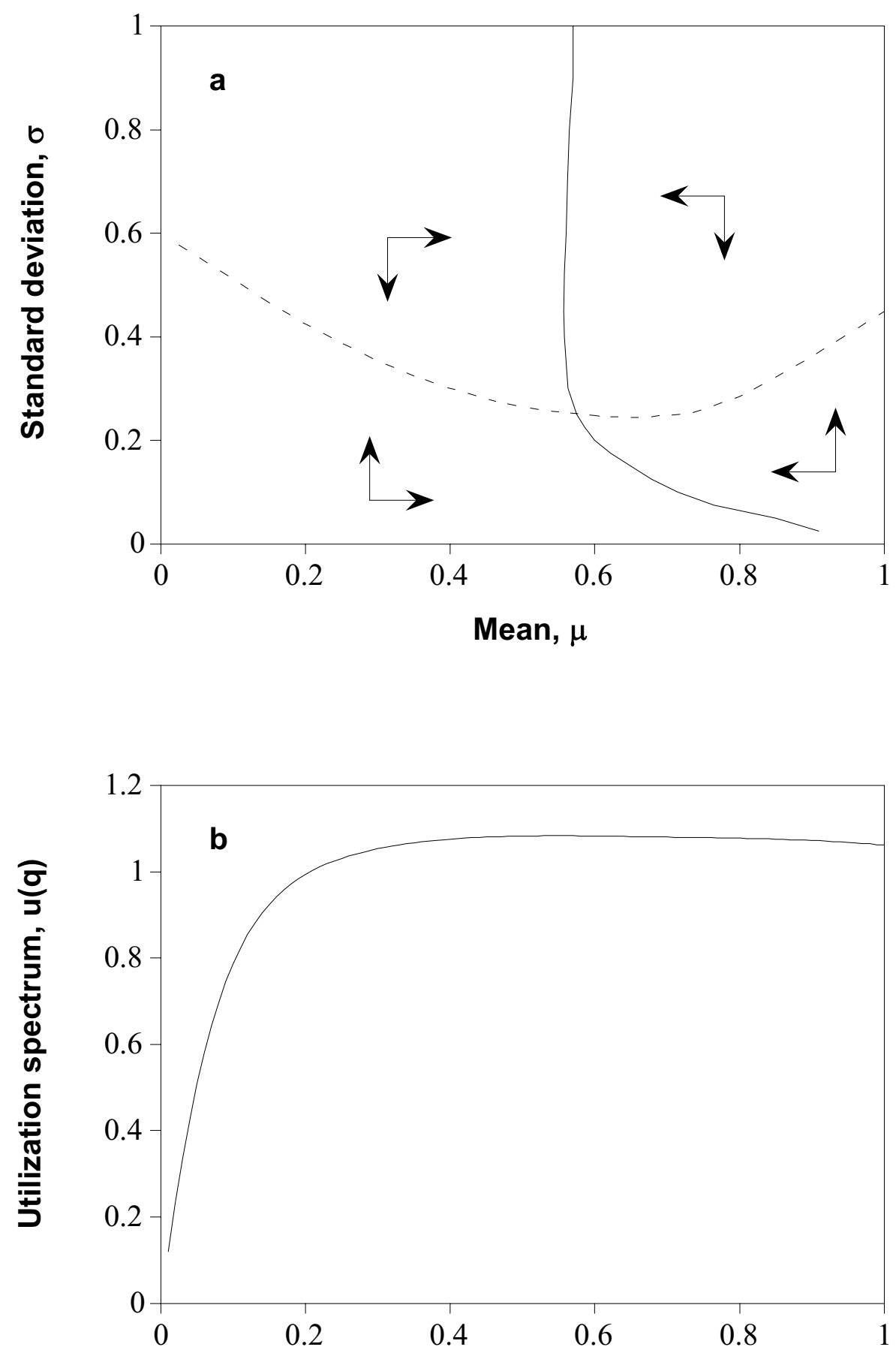

Plant quality, q 
Figure 5
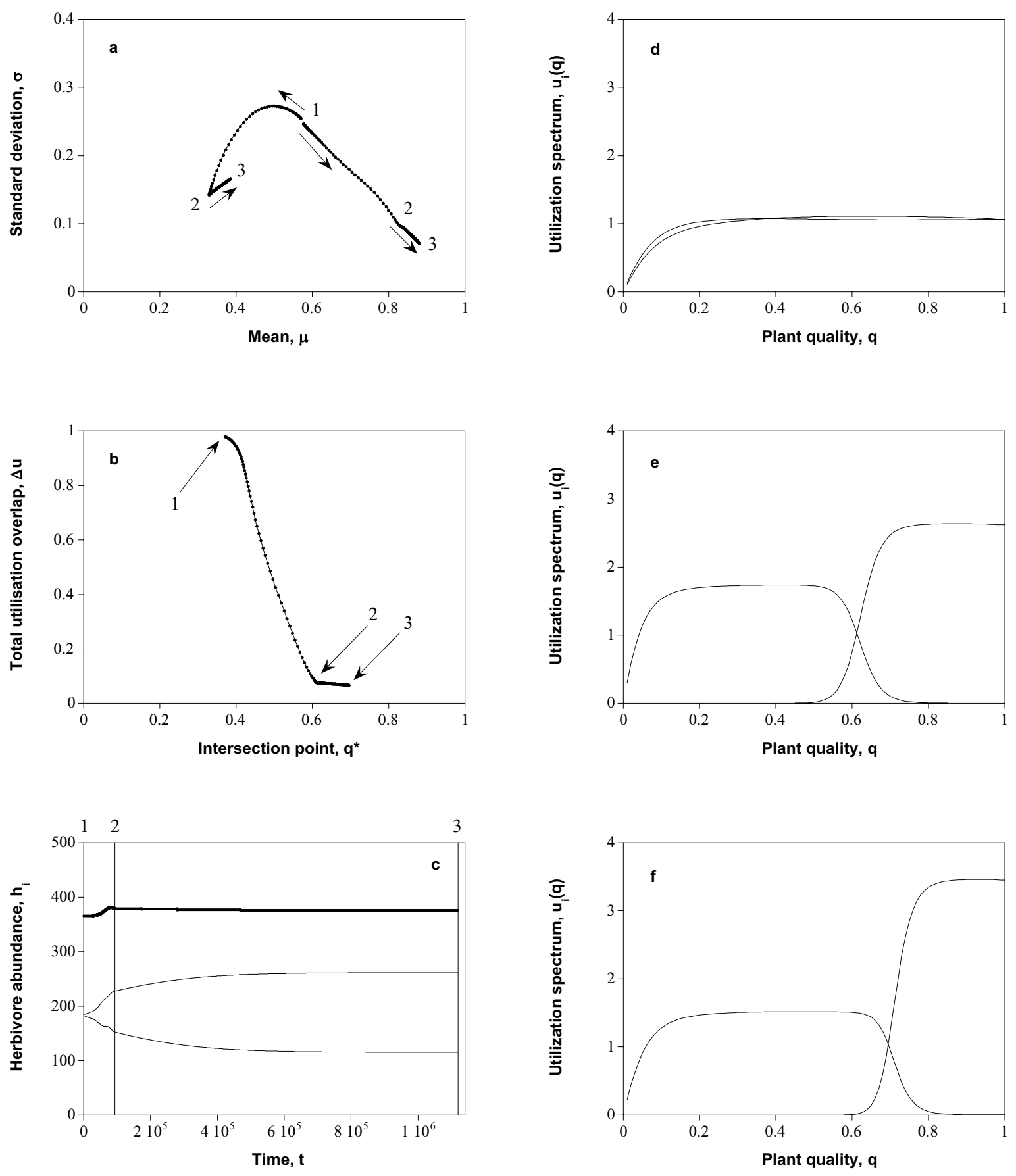
Figure 6
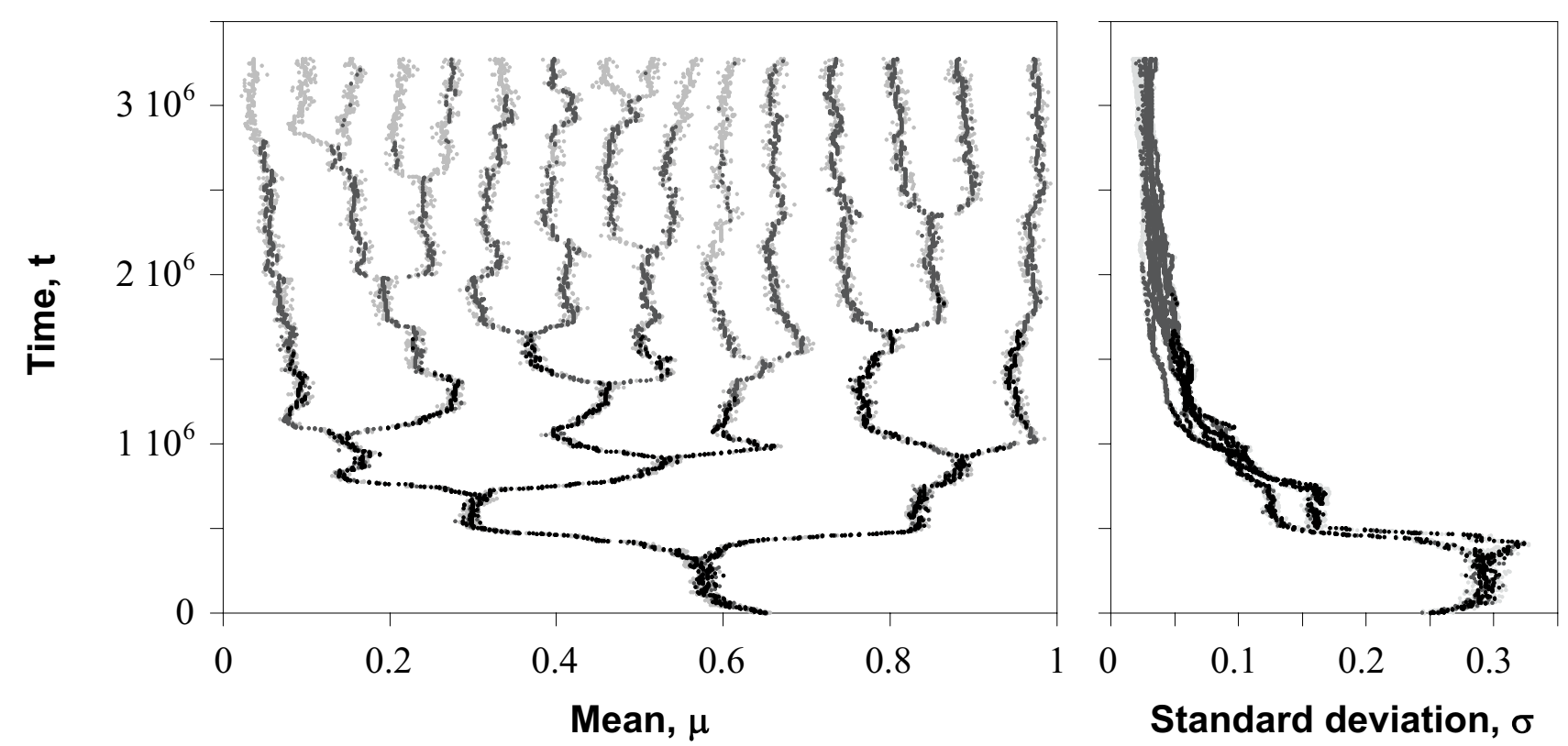
Figure A1

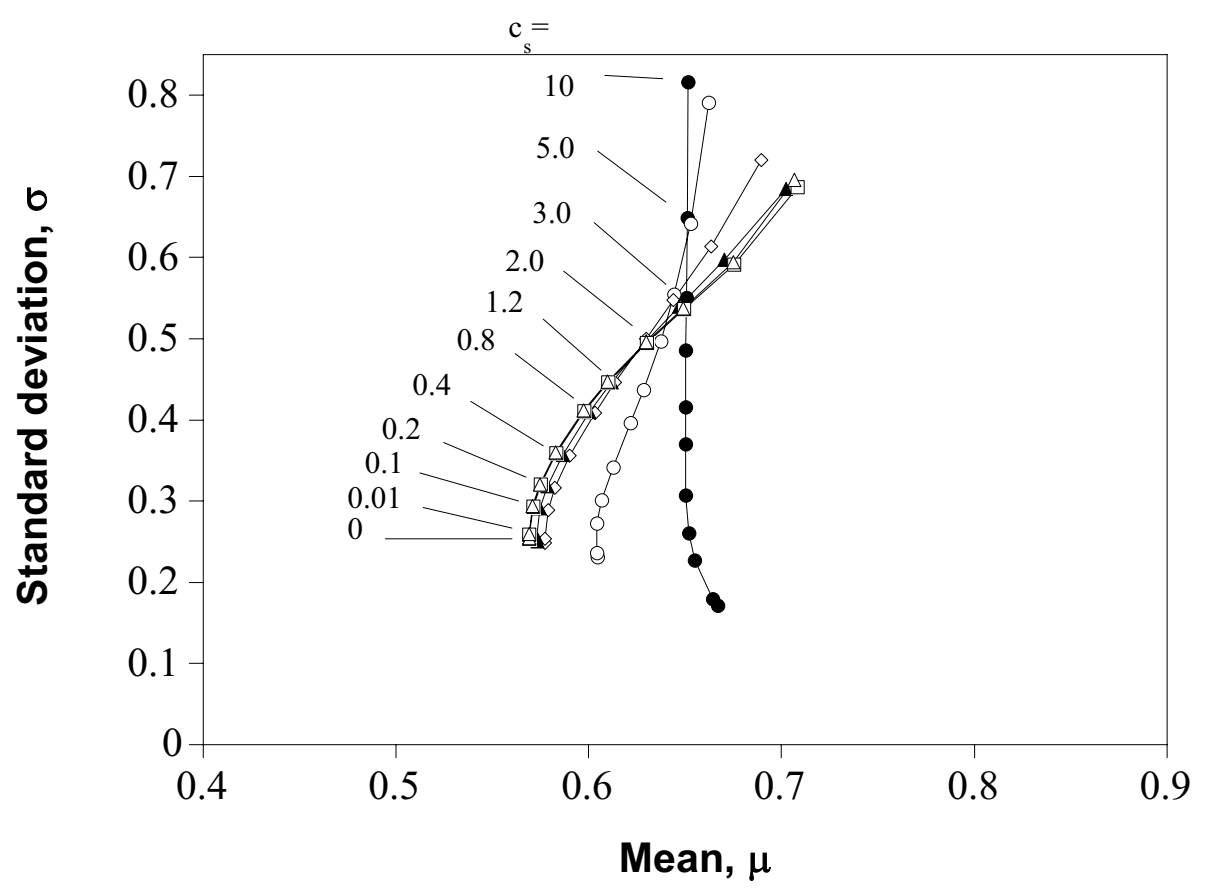


Figure A2

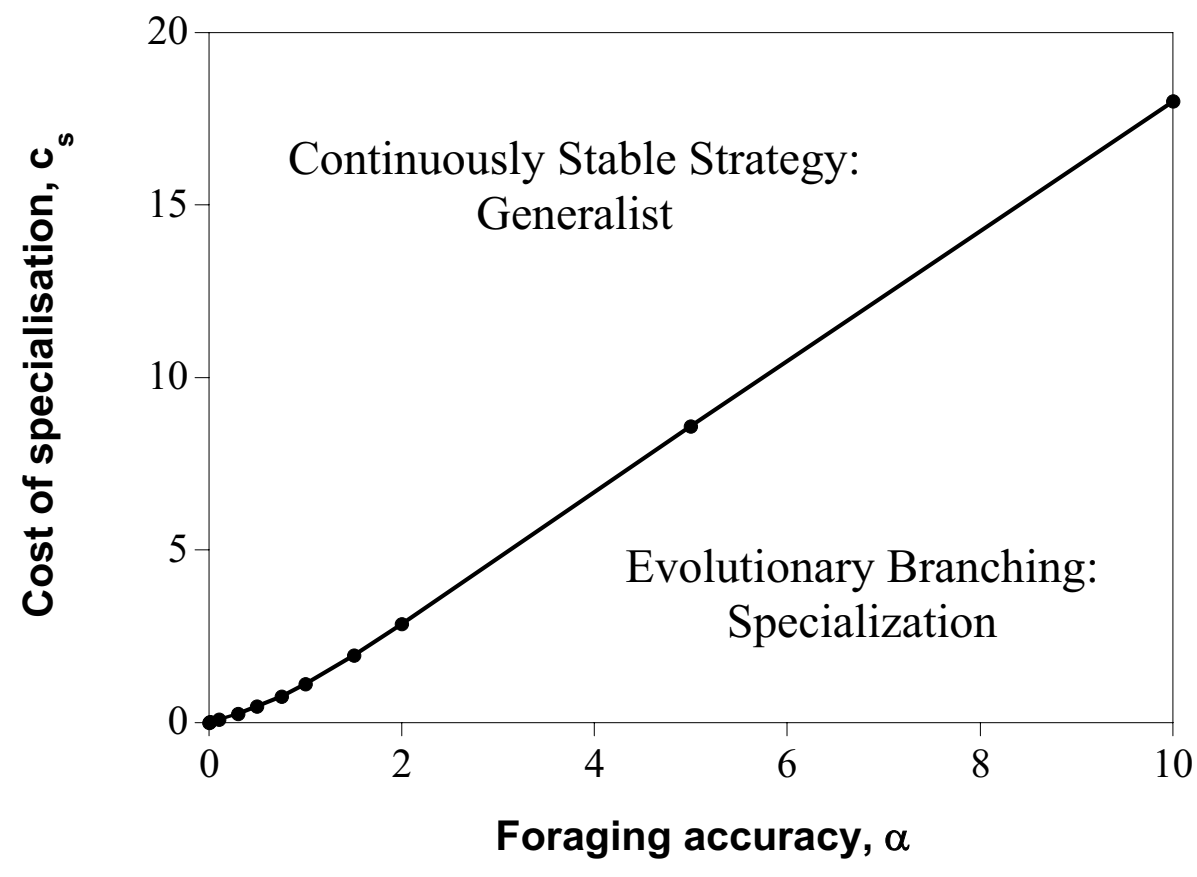


Figure A3
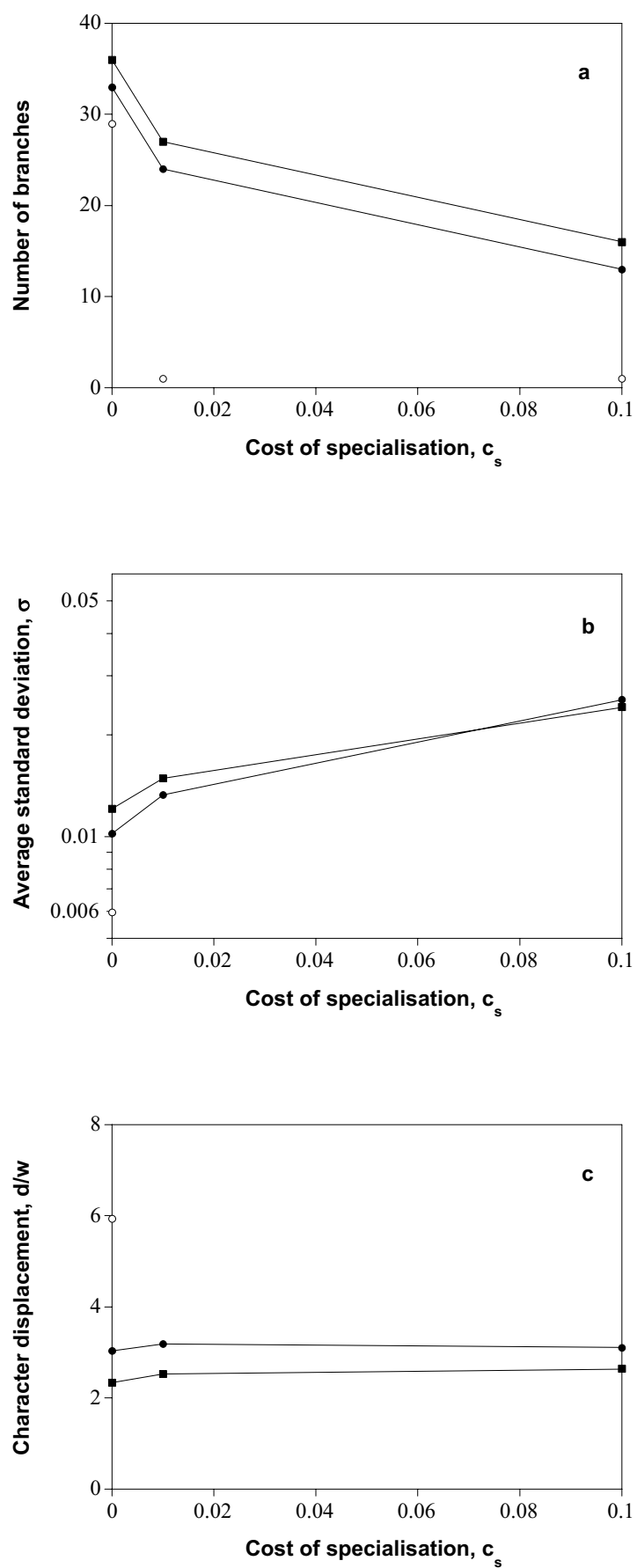